\title{
Microstructure and Property Modifications of Cold Rolled IF Steel by Local Laser Annealing
}

\author{
HÅKAN HALLBERG, FRÉDÉRIC ADAMSKI, SARAH BAÏZ, \\ and OLIVIER CASTELNAU
}

\begin{abstract}
Laser annealing experiments are performed on cold rolled IF steel whereby highly localized microstructure and property modification are achieved. The microstructure is seen to develop by strongly heterogeneous recrystallization to provide steep gradients, across the submillimeter scale, of grain size and crystallographic texture. Hardness mapping by microindentation is used to reveal the corresponding gradients in macroscopic properties. A 2D level set model of the microstructure development is established as a tool to further optimize the method and to investigate, for example, the development of grain size variations due to the strong and transient thermal gradient. Particular focus is given to the evolution of the beneficial $\gamma$-fiber texture during laser annealing. The simulations indicate that the influence of selective growth based on anisotropic grain boundary properties only has a minor effect on texture evolution compared to heterogeneous stored energy, temperature variations, and nucleation conditions. It is also shown that although the $\alpha$-fiber has an initial frequency advantage, the higher probability of $\gamma$ nucleation, in combination with a higher stored energy driving force in this fiber, promotes a stronger presence of the $\gamma$-fiber as also observed in experiments.
\end{abstract}

DOI: $10.1007 / \mathrm{s} 11661-017-4210-\mathrm{y}$

(C) The Author(s) 2017. This article is an open access publication

\section{INTRODUCTION}

WITH few exceptions, the present use of laser annealing is related to modification of the structure and properties of thin films. Examples include performance optimization of thin film cathodes in lithium-ion batteries, ${ }^{[1]}$ improvement of the properties of thin films for photovoltaic applications, ${ }^{[2]}$ fabrication of thin film diodes, ${ }^{[3]}$ and control of grain growth in thin films. ${ }^{[4]}$ Studies on laser annealing of larger specimens are scarcer. One example related to local modification of a thin Ni-Ti shape memory alloy strip with $0.5 \mathrm{~mm}$ thickness is given in Reference 5 and surface laser annealing of stainless steel is studied in References 6 and 7. Even less attention is given to the possibility of using laser annealing on larger samples of rolled sheet metal in order to manufacture functionally graded materials through local modifications of the microstructure, which is not achievable by standard annealing methods. This kind of applications are in focus of the present study.

HÅKAN HALLBERG is with the Division of Solid Mechanics, Lund University, P.O. Box 118, 22100 Lund, Sweden, and also with the Lab Process and Engineering in Mechanics and Materials (PIMM-UMR 8006), ENSAM, CNRS, CNAM, Hesam, 151 Bd de l'Hôpital, 75013 Paris, France. Contact e-mail: hakan.hallberg@solid.lth.se FRÉDÉRIC ADAMSKI, SARAH BAÏZ, and OLIVIER CASTELNAU are with the Lab Process and Engineering in Mechanics and Materials (PIMM-UMR 8006), ENSAM, CNRS, CNAM, Hesam.

Manuscript submitted January 11, 2017.

Article published online July 17, 2017
The method will be possible to apply in all areas of sheet metal modifications, such as deep drawing and folding as well as stamping, blanking, and punching. It is envisaged that the method will permit reduced tool forces and tool wear, and less risk of fracture and that finer geometrical tolerances can be met.

By local laser annealing of the material, a grain size gradient will develop in the microstructure and alter macroscopic material properties accordingly. As described by the classical Hall-Petch relation, the macroscopic yield stress of the material is related to the average grain size, and since the grain size can be altered by annealing - in the irradiated zone - laser annealing can be used to modify the yield stress of the material in selected regions. The laser annealing process also causes a reduction of the stored energy in the material by growth of new grains from (nearly) dislocation-free recrystallization nuclei. In addition, annealing also provides a characteristic recrystallization texture. In the case of the interstitial free (IF) steel, presently under consideration, cold rolling yields a texture dominated by two components comprising the $\alpha$-fiber, having the $\langle 110\rangle$ orientations parallel to the rolling direction (RD), and the $\gamma$-fiber having the $\langle 111\rangle$ orientations parallel to the normal direction (ND). In Reference 8, the initial fractions of the $\alpha$ - and $\gamma$-fibers after 75 pct cold rolling are found to be 41 and 27 pct, respectively, clearly showing the texture to be dominated by these components. Similar observations are made in Reference 9 
where, directly following 90 pct cold rolling, the volume fractions of the $\alpha$ - and $\gamma$-fibers are found to be 50 and 40 pct, respectively. The ratio between the fractions is, however, observed to change during subsequent annealing as the $\gamma$-fiber grows at the expense of the $\alpha$-fiber during recrystallization. The increased presence of the $\gamma$ fiber is also observed in References 10 and 11 and is of primary concern in practical applications as it significantly enhances the ductility and hence the formability of the material. ${ }^{[12-15]}$

The development of the characteristic texture components has been explained in terms of oriented nucleation and/or selective growth during recrystallization, ${ }^{[9,11,13,16-19]}$ or as size or frequency advantage for grains with $\gamma$ orientations. ${ }^{[20]}$ Preferential nucleation of grains with orientations belonging to the $\gamma$-fiber during annealing of IF steel is noted in a number of studies, for example in References 8, 9, 17, 19 through 22. The conditions under which the recrystallization texture develops are addressed in the numerical simulations in the present work along with the evolution of grain structure gradients.

In the present work, laser annealing experiments are performed on cold rolled IF steel. It is shown that local microstructural modifications through recrystallization and grain growth can be achieved and related macroscopic properties such as ductility and hardness can be modified in confined regions of the material. This makes laser annealing a promising method for manufacturing of functionally graded materials. To further optimize the method, additional studies on the correlation between processing conditions and microstructure evolution are required. This is addressed in the present work by establishing a level set-based numerical model in order to study the key mechanisms behind microstructure evolution during laser annealing. The model captures the central aspects of microstructure development at the mesoscale, while also considering the transient thermal gradient conditions which are present due to the laser processing.

Modeling of rolling and recrystallization textures in IF steel - considering oriented growth relative to oriented nucleation - is performed using a statistical model in References 11 and 23. But such an approach provides little information on the microstructure morphology or on the influence of any thermal gradient conditions. As an alternative, a number of different numerical techniques are available for performing mesoscale simulations of grain structure evolution in polycrystals. These include, for example, cellular automata algorithms, Monte Carlo Potts models, phase field models, and level set formulations. Considering applications involving IF steel, Monte Carlo Potts models are used in References 24 and 25 and a cellular automata formulation in Reference 14 to study microstructure evolution in this material, although not related to laser annealing and under isothermal conditions. In the present work, a mesoscale model of microstructure evolution is formulated in terms of a representative volume element (RVE), based on a level set formulation which is solved in a finite element setting. The level set method was introduced in Reference 26 and provides a framework for tracing the evolution of interfaces in different physical settings and the method has been employed in studies on grain growth, ${ }^{[27-31]}$ primary static recrystallization, ${ }^{[32]}$ and on dynamic recrys- tallization, ${ }^{[33,34]}$ A review of different methods for mesoscale modeling of recrystallization is provided in Reference 35. Regardless of which modeling approach is adopted, it is emphasized in, for example, Reference 36 that in order to be able to predict recrystallization textures in simulations, both a correct description of grain growth as well as a nonrandom nucleus texture are required. Both of these aspects are taken into account here.

Grain growth in the presence of a thermal gradient has been studied in numerical models previously, for example by Monte Carlo Potts and phase field models related to welding in References 37 and 38, respectively, and by Monte Carlo Potts modeling of $\mathrm{UO}_{2}$ nuclear fuel pellets in Reference 39. Similar to welding, the present local laser annealing is characterized by a rapidly varying and steep transient thermal gradient which is accounted for in the numerical simulations. To provide the spatial and temporal temperature variation during laser annealing, a standard thermal finite element model of the macroscopic annealing process is considered initially. This permits the temperature gradients in the material to be identified for subsequent employment in the microstructure RVEs, which are smaller subdomains of the macroscopic specimen. Use of level set-based RVEs in such modeling framework has not been considered previously in the literature.

This paper is structured into sections, beginning with Section II, discussing the laser annealing experiments. To provide the spatial and temporal temperature history in the workpiece during laser irradiation, the thermal finite element model is introduced in Section III. The numerical simulations of microstructure evolution are performed using a level set formulation which is established in Section IV, with subsections providing details of recrystallization and grain growth modeling. The details of the adopted simulation model are presented in Section V, followed by a presentation of the results and a discussion in Section VI. Some concluding remarks close the paper in Section VII.

\section{LASER ANNEALING EXPERIMENTS}

The experiments were performed on a Ti-stabilized IF steel sheet which had been cold rolled from an initial thickness of $4.5 \mathrm{~mm}$ down to a final thickness of $1.263 \mathrm{~mm}$, resulting in a 72 pct thickness reduction. The average grain size in the cold rolled material was $59 \mu \mathrm{m}$ along RD, $8.7 \mu \mathrm{m}$ along $\mathrm{ND}$, and $31.7 \mu \mathrm{m}$ along the transverse direction (TD), similar to what was found in Reference 40 for ultralow carbon steel at a corresponding thickness reduction.

Specimens measuring approximately $100 \times 100 \mathrm{~mm}^{2}$ were taken from the rolled sheet for the experiments. A Nd-YAG laser source with $1.06 \mu \mathrm{m}$ wavelength was used to provide a square $12 \times 12 \mathrm{~mm}^{2}$ beam cross-section with uniform "top-hat" distribution of the laser intensity. Different laser powers and irradiation times were investigated in order to find the processing conditions which provided appropriate annealing temperatures without melting the material. For the present study, the results obtained with a laser power of $P=320 \mathrm{~W}$ and an 
irradiation time of $9 \mathrm{~s}$ are considered. To measure the evolution of the thermal field, thermocouples were spot welded to the bottom surface of the specimen, facing away from the laser beam. The temperature measurements are used to calibrate the thermal finite element model in Section III. Further details on the experimental procedure and the influence of laser process parameters on the obtained microstructure will be published elsewhere.The microstructure obtained here was investigated before and after laser annealing using a combination of optical microscopy, $c f$. Figure 1, and EBSD mapping, $c f$. Figure 2. A pronounced gradient in the grain size was obtained, as shown in Figure 1. Figure 1 also shows how the cold rolled laminar grain structure is gradually replaced by a recrystallized grain structure of nearly equiaxed grains in the annealed region. The grain size gradient can also be seen in Figure 3, showing the variation of the average grain size across the laser irradiated zone. It can be noted from Figure 3 that the grain size is slightly larger at the center of the annealed zone compared to at the edges of the zone. This is most likely due to that the temperature has been higher closer to the laser beam center which has permitted more growth of the recrystallized grains than in the less heated regions at the edges of the annealing zone.Following the variations in grain size in the workpiece, the corresponding gradients in macroscopic material properties were also measured by mapping of the Vickers hardness in a region around the focal point of the laser beam by means of microindentation, $c f$. Figure 4 . It can be seen that the laser annealing has resulted in a significant decrease of hardness at the center of the laser irradiated zone.Looking at the EBSD maps in Figure 2, obtained in the RD-TD plane on the top surface of the specimen, the area fractions of the two dominant texture fibers after annealing can be distinguished. An angular tolerance of $\pm 13.5 \mathrm{deg}$ around the ideal orientations was used to identify the fiber components. Red and blue colors indicate $\alpha$ - and $\gamma$-grains, respectively. Figure 2(a) shows an EBSD map at point A, close to the laser center, where the $\alpha$ - and $\gamma$-fractions are 17.7 and 31.1 pct, respectively. Figure 2(b) shows an EBSD map at point B, located further away from the laser center, where the $\alpha$ and $\gamma$-fractions are found to be 20.4 and 29.4 pct, respectively. It is evident that the $\gamma$-fiber dominates over the $\alpha$-fiber after recrystallization, as also reported in the literature, for example in References 9 and 11. This trend becomes increasingly pronounced closer to the laser beam centerline, i.e., deeper into the region which has been exposed to the highest annealing temperatures.

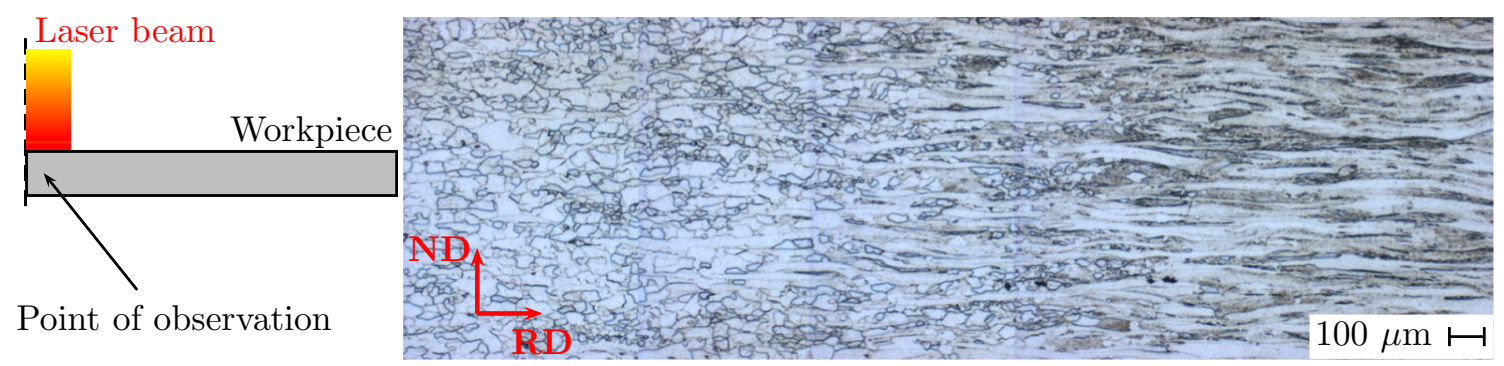

Fig. 1-Microstructure in the RD-ND plane at the point indicated. A clear gradient in grain size and structure has developed due to the laser annealing.

(a) Point A

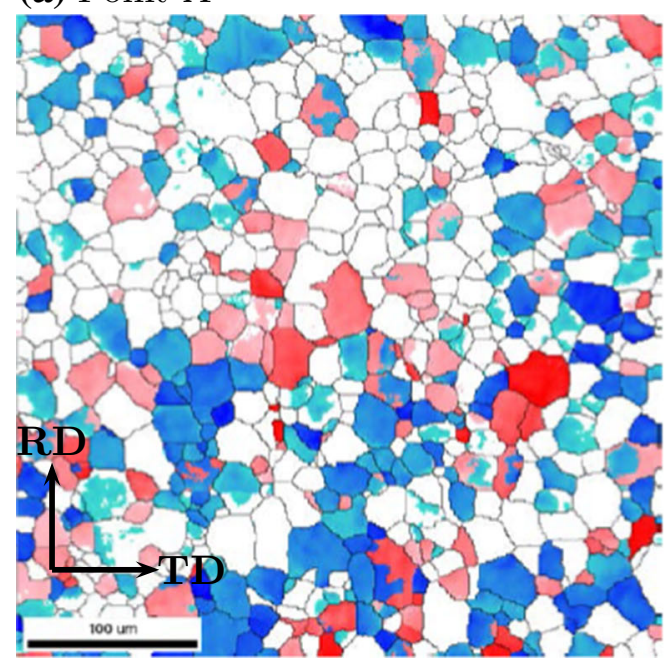

(b) Point B

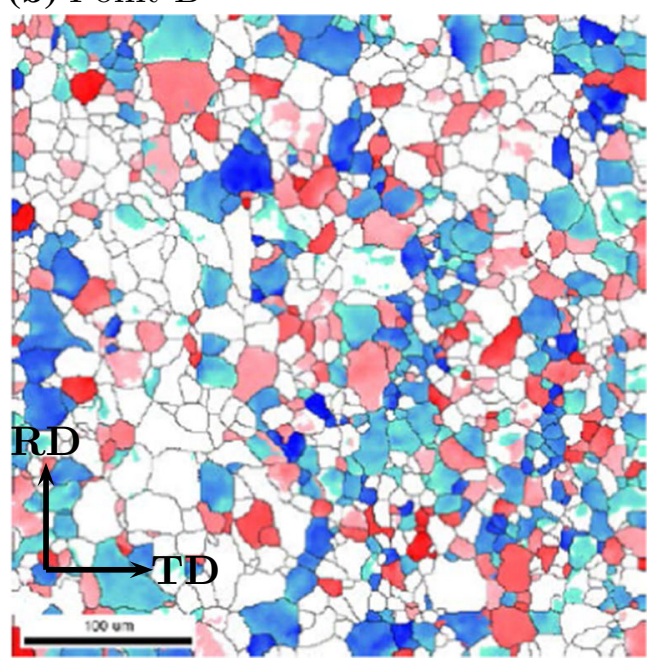

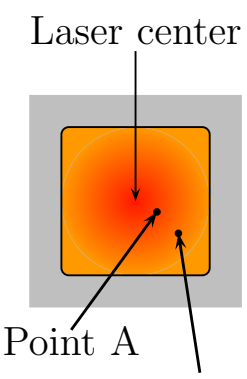

Point B
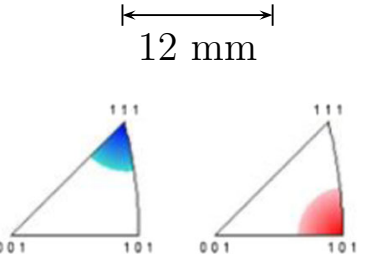

Fig. 2-Presence of the $\alpha$-fiber (red) and $\gamma$-fiber (blue) at the two locations indicated in the recrystallization zone, after laser annealing. The EBSD maps are made at the top surface of the specimen, looking down along the ND direction. (a) Point A, located close to the laser center. (b) Point $\mathrm{B}$, located further away from the laser center (Color figure online). 


\section{THERMAL MODEL}

The temporal and spatial evolution of the thermal field in the workpiece due to laser irradiation is modeled using the heat equation:

$$
\rho c \dot{T}+\operatorname{div}(\boldsymbol{q})-Q=0,
$$

where $T$ is the absolute temperature. Further, $\rho=7870 \mathrm{~kg} / \mathrm{m}^{3}$ and $c=432 \mathrm{~J} / \mathrm{kg} / \mathrm{K}$ are the mass density and specific heat, respectively. A superposed dot indicates derivation with respect to time. Also found in Eq. [2], $\boldsymbol{q}=-k \nabla T$ denotes the heat flux vector as given by Fourier's law, with $k=88 \mathrm{Wm}^{-1} \mathrm{~K}^{-1}$ being the isotropic thermal conductivity. The quantity $Q$ represents any internal heat source, such as the latent heat associated with phase transformations. ${ }^{[41]}$ In the present case, however, the material remains in the solid state at all times and it is assumed that $Q=0$. The en-

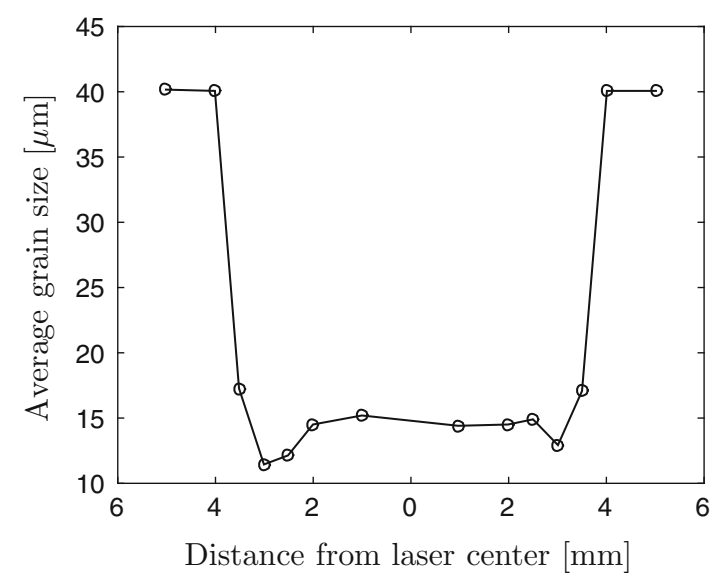

Fig. 3-Variation of the average grain size across the laser irradiated zone. ergy input from the laser irradiation is modeled as a surface heat flux $q_{l}$, normal to the surface. The square laser beam profile with a uniform intensity distribution which was used in the experiments, can be modeled by the heat flux

$$
q_{l}(r)=\left\{\begin{array}{ccc}
(1-R) \frac{P}{r_{l}^{2}} & \text { if } & r \leq r_{l} \\
0 & \text { if } & r>r_{l}
\end{array},\right.
$$

where $R$ is the surface reflectivity, estimated to be 0.5 in the model, $P$ the laser beam power, and $r_{l}=12 \mathrm{~mm}$ is the laser beam width. The format of the laser heat flux in Eq. [2] is also considered in, e.g. References 41 through 43. A schematic illustration of the thermal model is shown in Figure 5, also defining the coordinate $r$. Convective boundary conditions are prescribed along the section of the top surface which is not exposed to laser irradiation as well as along the right and bottom boundaries. The convective flux boundary conditions apply normal to the surfaces and have the form $q_{c}=\alpha\left(T-T_{\infty}\right)$, where $\alpha=350 \mathrm{~W} / \mathrm{m}^{2} / \mathrm{K}$ is the convection coefficient and $T_{\infty}=298 \mathrm{~K}\left(25^{\circ} \mathrm{C}\right)$ is the ambient (room) temperature, also set as the initial temperature in the sample. Advantage is taken of the vertical symmetry plane, indicated in Figure 5. The dimensions of the model are $w=50 \mathrm{~mm}$ and $h=1.263 \mathrm{~mm}$, as for the real specimen.

The thermal model is solved in a $2 \mathrm{D}$ finite element setting and the temperature evolution at the points $\mathrm{A}-\mathrm{C}$ in Figure 5 is traced throughout the solution. These points correspond to the locations of the thermocouples which were used during the experiments. Figure 6(a) shows the temperatures at the three points as obtained in the simulation (solid lines) compared to the temperatures registered during the experiment (symbols). It can be noted that the temperature rises rapidly during the initial laser irradiation, and a sharp peak occurs at the point in time when the laser is switched off (after 9 seconds) and the specimen is allowed to cool.

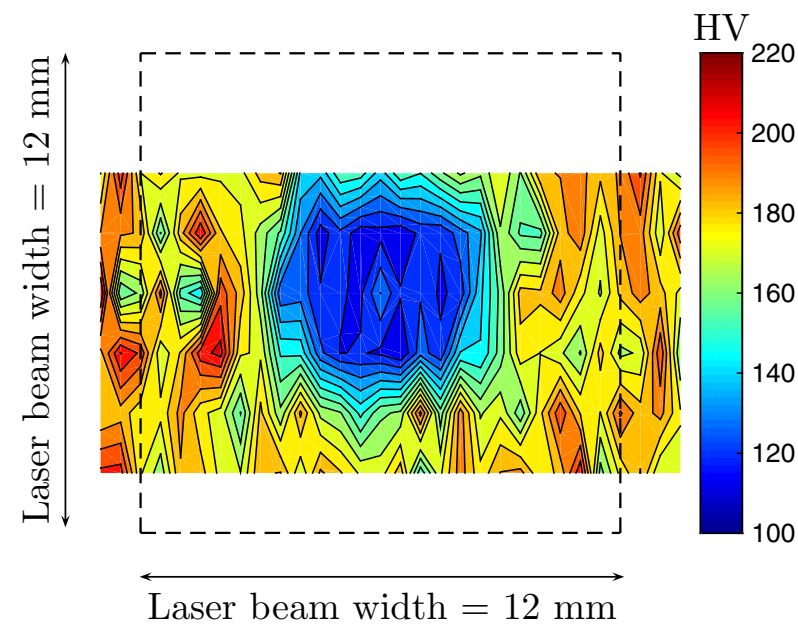

(a)

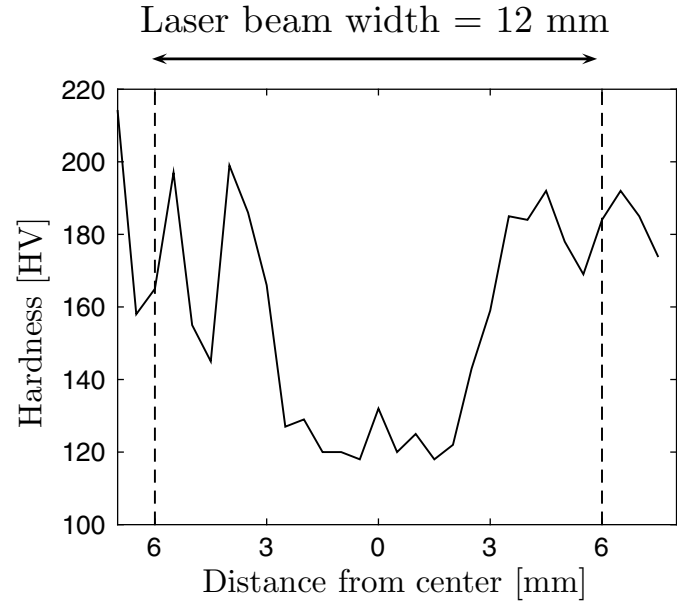

(b)

Fig. 4 - (a) Map of the Vickers hardness, measured by microindentation, over the specimen surface in a region surrounding the laser beam center. (b) Hardness variation along a line through the laser center. 
Considering Figures 3 and 4, it is seen that the most significant influence of the laser annealing is found within a region of $4 \mathrm{~mm}$ from the laser center, in the present case. Three locations in the macroscopic specimen are considered for the microstructure simulations, indicated by the microstructure representative volume elements (RVEs) shown in Figure 5. The RVEs are located along the mid-section of the workpiece at the distances $r=0, r=2 \mathrm{~mm}$, and $r=4 \mathrm{~mm}$ from the laser beam center, respectively (measured to the left edges of the RVEs). Each RVE has the dimensions $800 \times 200$ $\mu \mathrm{m}^{2}$. Using different locations allows investigation of the influence of the severity of the thermal gradient and to obtain the annealed microstructure at different distances away from the laser center.

Due to the limited thickness of the specimen, there are only very small variations in the temperature distribution along the normal direction (ND), $c f$. Figure 5. Neglecting these small variations, the microstructure RVEs are assumed to be subject to temperature gradients along the RD direction only. Further, as the RVEs are of very small dimensions, this temperature variation can be assumed to be linear without any appreciable loss of precision. Hence, it is sufficient to record the temperature history at the left and right edges of the individual microstructure RVEs during the thermal simulation, and subsequently use each of these pairs of time-varying data to obtain the temperature at any point in each of the RVEs through linear interpolation at any time during the annealing process. The temperatures from the thermal model at the left and right edges of the individual RVEs are shown in Figure 6(b). It can be noted that the maximum temperature variation across any of the RVEs occurs at the end of the irradiation phase, just as the laser is switched off and the cooling phase begins.

\section{LEVEL SET MODELING OF RECRYSTALLIZATION AND GRAIN GROWTH}

A level set approach is taken to modeling of the microstructure evolution and advantage is taken of the framework previously established in References 31 and 33. The main features of the level set formulation are summarized here for completeness and in order to

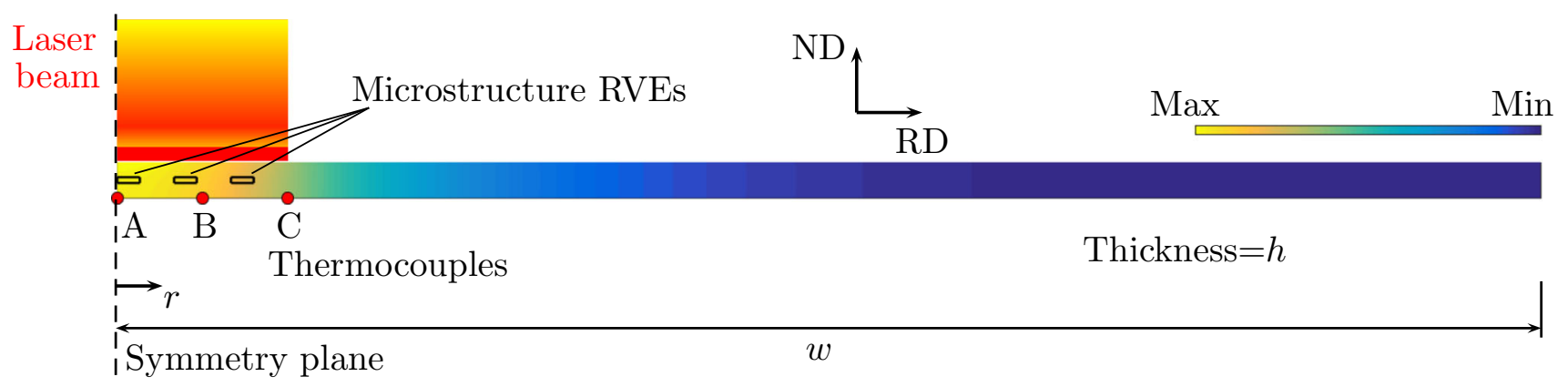

Fig. 5-Schematic illustration of the 2D thermal model of the laser annealing process (not drawn to scale). Points A-C correspond to the placement of thermocouples during the experiments. Note that advantage is taken of symmetry along the left boundary, where $r=0$.

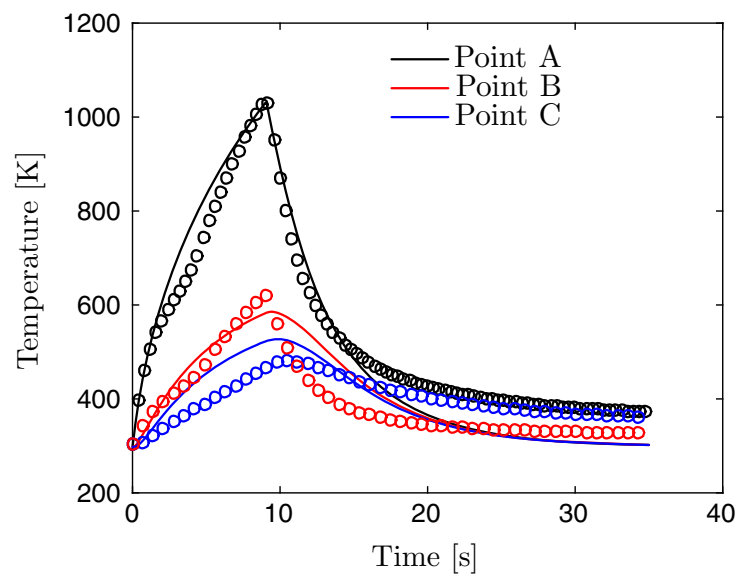

(a)

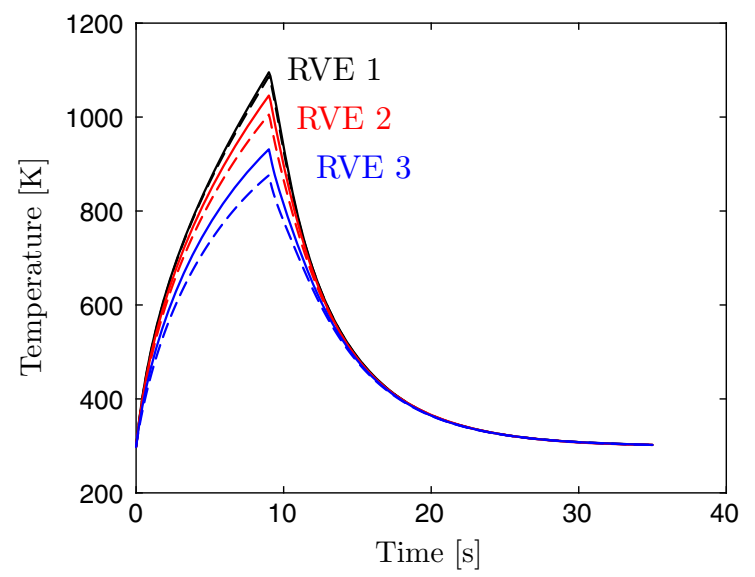

(b)

Fig. 6-(a) Temperature history at the points A-C, shown in Fig. 5. The solid lines show the simulated temperature evolution and the symbols are values measured during the experiments. (b) Temperature variation at the left and right edges, indicated by solid and dashed lines, respectively, of the microstructure RVEs, $c f$. Fig. 5. 
identify quantities that are central to the present study. For additional details on the level set formulation and the numerical implementation, please refer to Reference 33.

Central to the level set method is the introduction of a scalar field $\phi(\boldsymbol{x}, t)$, i.e. the level set function, defined throughout the computational domain $\Omega$. Here, $x$ and $t$ were introduced to denote the spatial coordinates and the time, respectively. The zero contour, where $\phi=0$, represents the spatial discontinuity $\Gamma$ which in the present physical settings traces the location of a grain boundary in the microstructure. The function $\phi$ is taken to be a distance function, meaning that the value of $\phi$ at a certain point $\boldsymbol{x}$ corresponds to the closest distance $d(\boldsymbol{x}, t, \Gamma)$ between the point and the interface at the current time. In addition, a sign convention is respected whereby $\phi$ is defined to be positive inside the domain delimited by the interface, zero at the interface, and negative elsewhere. These preliminaries can be stated as

$$
\left\{\begin{array}{c}
\phi(\boldsymbol{x}, t)=d(\boldsymbol{x}, t, \Gamma), \boldsymbol{x} \in \Omega \\
\Gamma=\{\boldsymbol{x} \in \Omega, \phi(\boldsymbol{x})=0\} .
\end{array}\right.
$$

Since $\phi(\boldsymbol{x}, t)$ is defined as a signed distance function, it holds that

$$
\|\nabla \phi(\boldsymbol{x}, t)\|=1, \quad \boldsymbol{x} \in \Omega .
$$

The local interface normal $\boldsymbol{n}$ and curvature $\kappa$ can be evaluated directly from the level set function by considering

$$
\left\{\begin{array}{l}
\boldsymbol{n}=\frac{\nabla \phi}{\|\nabla \phi\|} \equiv \nabla \phi, \\
\kappa=\nabla \boldsymbol{n} \equiv \nabla\left(\frac{\nabla \phi}{\|\nabla \phi\|}\right) \equiv \nabla^{2} \phi
\end{array}\right.
$$

where the last equalities hold as long as the property $\|\nabla \phi\|=1$ is valid. During the numerical solution procedure, the level sets may occasionally overlap at interfaces or separate to create artificial voids. This requires a level set interaction correction to be performed. In addition, there is sometimes a tendency for the level sets to drift from being proper distance functions. Adopting the standard approach, the latter issue is in the present work remedied by performing a reinitialization of the level set functions in each time step, whereby $\|\nabla \phi\|=1$ is ensured. Level set interaction correction and reinitialization are in the present implementation performed as described in Reference 33.

Next, differentiation of Eq. [3] with respect to time provides the evolution law for the interface as

$$
\frac{\partial \phi}{\partial t}+(\nabla \phi) \frac{\partial \boldsymbol{x}}{\partial t}=0 .
$$

To generalize the result in Eq. [6] to situations with an arbitrary number of interfaces, a total number of $N_{\phi}$ level set functions are employed, allowing Eq. [6] to be stated as

$$
\left\{\begin{array}{l}
\frac{\partial \phi_{i}}{\partial t}+\boldsymbol{v}^{T} \nabla \phi_{i}=0, \\
\phi_{i}(t=0, \boldsymbol{x})=\phi_{i}^{0}(\boldsymbol{x}),
\end{array} \quad \forall i \in\left\{1 \ldots N_{\phi}\right\},\right.
$$

where $\boldsymbol{v}=v_{n} \boldsymbol{n}$ was introduced to denote the local interface velocity vector with magnitude $v_{n}$. In addition, $\phi_{i}^{0}(\boldsymbol{x})$ represents the initial positions of the individual interfaces at time $t=0$.

\section{A. Level Sets Applied to Recrystallization Modeling}

Cold working of metallic materials increases the stored energy, most significantly through an increased dislocation density in the crystal structures. The raised internal energy leaves the material in a thermodynamic non-equilibrium state and recrystallization is one of the main mechanisms to reduce the stored energy. Recrystallized grains may grow by migration of high-angle grain boundaries to consume the high-energy cold worked matrix material and the local grain boundary velocity $\boldsymbol{v}$ can be written as

$$
\boldsymbol{v}=m p \boldsymbol{n},
$$

where $m$ is the boundary mobility and $p$ is the driving pressure acting on the grain boundary. ${ }^{44]}$ This driving pressure may be due to a number of different factors but the two main components are

$$
\begin{aligned}
& p_{\rho}=\tau \llbracket \rho \rrbracket \\
& p_{\gamma}=-\kappa \gamma
\end{aligned},
$$

where $p_{\rho}$ is due to a dislocation density jump $\llbracket \rho \rrbracket$ across the interface, with the dislocation line tension given by $\tau=\mu b^{2} / 2$. The magnitude of the Burgers vector and the shear modulus are denoted by $b$ and $\mu$, respectively. In addition, $\gamma$ is due to the curvature of the boundary and the grain boundary energy $\gamma$. The total driving pressure acting on a grain boundary can be stated as

$$
p=p_{\rho}+p_{\gamma} .
$$

Working in the level set framework outlined in Section IV, the local grain boundary velocity in Eq. [8] can be written as

$$
\boldsymbol{v}=\boldsymbol{v}_{\rho}+\boldsymbol{v}_{\gamma}
$$

where the following quantities were introduced:

$$
\begin{array}{cc}
\boldsymbol{v}_{\rho}=v_{\rho} \boldsymbol{n}, & v_{\rho}=m \tau \llbracket \rho \rrbracket \\
\boldsymbol{v}_{\gamma}=v_{\gamma} \kappa \boldsymbol{n}, & v_{\gamma}=-m \gamma .
\end{array}
$$

Noting that the interface normal $\boldsymbol{n}$ and the interface curvature $\kappa$ can be obtained directly from the level set formulation according to Eq. [5], the velocity components in Eq. [12] can be employed together with Eq. [7] to obtain

$$
\left\{\begin{array}{l}
\frac{\partial \phi_{i}}{\partial t}+v_{\rho}^{T} \nabla \phi_{i}+v_{\gamma} \nabla^{2} \phi_{i}=0, \quad \forall i \in\left\{1 \ldots N_{\phi}\right\} . \\
\phi_{i}(\boldsymbol{x}, t=0)=\phi_{i}^{0}(\boldsymbol{x}),
\end{array}\right.
$$

The transient advection/diffusion equation in Eq. [13] constitutes the level set formulation which is solved in a finite element setting in the present study. The convective velocity field $\boldsymbol{v}_{\rho}$ is evaluated according to the 
method introduced in Reference 32 in order to ensure a continuous velocity field. The adopted treatment of the convective velocity is also further discussed in Reference 33 .

\section{B. Nucleation}

The two fundamental aspects of microstructure transformation through recrystallization are nucleation and subsequent growth of new grains. As primary static recrystallization is under consideration here, nucleation can be expected to be a process of discontinuous subgrain growth. ${ }^{[4-47]}$

A successful nucleation event requires the local microstructure conditions to satisfy both a kinetic criterion, whereby sufficiently mobile grain boundaries should be formed by the nucleation, and a thermodynamic criterion, according to which enough stored energy (dislocation density) difference must be present across the interface to exert a positive drag force. Microstructure sites which satisfy these conditions are primarily found along grain boundaries but also, with lesser probability, in grain interiors at larger particle inclusions and along shear bands. ${ }^{[4]}$ Since subgrain structures are not spatially resolved in the present model formulation, it is assumed that the necessary dislocation substructures are present after the initial cold working, as indeed indicated in Reference 48, and that grain boundaries are the most likely nucleation sites, as noted for IF steel in, e.g., References 10 and 18.

In the present model, nucleation is modeled as site saturated. By this approach, a number of nuclei are distributed in the microstructure at the onset of the simulation. The number of nuclei is chosen to mimic the recrystallization behavior observed in the experimental results. Nucleation is in the present model assumed to take place at sites along the grain boundaries and the nuclei are randomly placed at such locations. In addition, different assumptions on nucleation in one or both of the $\alpha$ - and $\gamma$-fibers will be considered. This is further discussed in Section V.

Similar approaches to modeling of the nucleation phase as adopted here are also used in the cellular automata model of static recrystallization in IF steel in Reference 14 and in the Monte Carlo Potts models in References 24 and 25. Site-saturated nucleation of primary static recrystallization is also adopted in the Monte Carlo Potts models in References 49 and 50. The details on modeling the nucleation event in a finite element-based level set formulation are provided in Reference 33.

New recrystallization nuclei are added in the present model by identifying a nodal point in the finite element mesh where the nucleation criteria are met and subsequently adding a circular contour, i.e., a new level set function, centered around that node. The radius of the new zero contour corresponds to the radial size of the nucleus. Being strain-free, new recrystallization nuclei will be added with zero initial dislocation density.

The present model is formulated in such a way that the nature of the nucleation event can be controlled in terms of orientation and regarding where the nucleation is allowed to take place. This means, for example, that nucleation can be controlled to take place in either or both of the populations of $\alpha$ - and $\gamma$-fiber grains. Different combinations of these nucleation conditions are investigated in the numerical simulations in Section V.

\section{Variation of Properties with Misorientation and Temperature}

The orientation of each grain is defined by the three Euler angles $\varphi_{1}, \Phi$, and $\varphi_{2}$ (following the Bunge convention), whereby the rotation matrix for each grain can be stated as $\boldsymbol{g}\left(\varphi_{1}, \Phi, \varphi_{2}\right)$. The misorientation between two crystals with orientations $\boldsymbol{g}_{i}$ and $\boldsymbol{g}_{j}$ is found as $\Delta \boldsymbol{g}_{i j}=\boldsymbol{g}_{j} \boldsymbol{g}_{i}^{T}$, providing the misorientation as the rotation which rotates one crystal reference frame into that of another crystal. Considering crystal symmetries, the minimum misorientation is evaluated from

$$
\theta=\min _{\boldsymbol{O}_{s} \in \mathscr{G}_{c}}\left|\operatorname{acos}\left[\frac{1}{2}\left\{\operatorname{tr}\left(\boldsymbol{O}_{\mathrm{s}} \Delta \boldsymbol{g}\right)-1\right\}\right]\right|
$$

where the indices $i$ and $j$ were dropped for convenience and where $\boldsymbol{O}_{s}$ is one of the 24 operators in the cubic symmetry group $\mathscr{G}_{c}$. The absolute value $|\cdot|$ is taken in Eq. [14] to reflect that a negative angle simply indicates that the rotation axis points in the opposite direction. In Eq. [14], $\operatorname{tr}(\cdot)$ denotes the trace of a tensor.

The grain boundary energy is in the present model allowed to vary with the local grain boundary misorientation according to the classical Read-Shockley relation:

$$
\gamma(\theta)= \begin{cases}\gamma_{m} \frac{\theta}{\theta_{m}}\left[1-\ln \left(\frac{\theta}{\theta_{m}}\right)\right] & \text { if } \theta<\theta_{\mathrm{m}} \\ \gamma_{m} & \text { if } \theta \geq \theta_{\mathrm{m}}\end{cases}
$$

where $\theta_{m}=15 \mathrm{deg}$ is the angle that differentiates between low- and high-angle boundaries and $\gamma_{m}$ is the energy of general high-angle grain boundaries. The misorientation dependence of the grain boundary energy is shown in Figure 7(a) (blue line). Parameters related to the grain boundary properties are provided in Table I.

Migration of misoriented grain boundaries can be viewed as a thermally activated process and the temperature dependence of the grain boundary mobility is given by

$$
m_{T}(T)=\frac{b^{2} D_{g b}}{k_{B} T} \quad \text { where } \quad D_{g b}=D_{0} \exp \left(-\frac{Q_{m}}{k_{B} T}\right),
$$

where $k_{B}$ is the Boltzmann constant and $D_{g b}$ is the grain boundary diffusivity with $D_{0}$ and $Q_{m}$ being the diffusion coefficient and the activation energy for grain boundary migration, respectively. The resulting temperature dependence of the grain boundary mobility is shown in Figure 7(b) (red line). The mobility can be seen to be significantly increased for temperatures above approximately $850 \mathrm{~K}\left(577^{\circ} \mathrm{C}\right)$, i.e., at temperatures which are reached locally in the material during the present laser annealing, $c f$. Figure 6. 
The mobility of low-angle boundaries, having $\theta \leq \theta_{m}$, is generally much lower than that of high-angle boundaries. Following Reference 46, a misorientation dependence $m_{\theta}$ of the mobility can be added to Eq. [16] to provide

$$
\begin{aligned}
& m(T, \theta)=m_{T}(T) m_{\theta}(\theta) \quad \text { where } \\
& { }_{\theta}(\theta)=\left[1-\exp \left(-5\left\{\frac{\theta}{\theta_{m}}\right\}^{4}\right)\right] .
\end{aligned}
$$

The misorientation dependence of the grain boundary mobility is illustrated in Figure 7(a) (red line).

Also the shear modulus, appearing in the dislocation line tension in Eq. [9], has a temperature dependence which needs to be taken into account as it influences the stored energy driving force for grain growth. The temperature dependence of the shear modulus for $\alpha-\mathrm{Fe}$ is stated in Reference 52 as

$$
\mu(T)=69.2 \times 10^{9}\left(1-1.31 \frac{T-300}{T_{m}}\right) \quad P a,
$$

where $T_{m}=1810 \mathrm{~K}\left(1537{ }^{\circ} \mathrm{C}\right)$ is the melting temperature. The format in Eq. [18] is adopted here and the variation of the shear modulus with temperature is shown in Figure 7(b) (blue line).

\section{Efficient Handling of Local Grain Boundary Properties in a Level Set Framework}

As grain boundary energy and mobility are local quantities, they have to be evaluated along each interface in the polycrystal. In addition, during grain growth, an individual grain may become adjacent to any other grain. As each of the grain boundary interfaces may comprise hundreds of grain boundary segments, depending on the degree of mesh refinement, and if the evaluation is done in every time step of the simulation, the computational effort in considering Eqs. [14], [15], and $m_{\theta}$ in [17] may be significant. It can also be noted that evaluation of Eq. [14] involves a minimization wherein several symmetry rotations have to be compared, and the effort depends on the order of crystal symmetry. However, as the anisotropy of the local grain boundary properties is presently described by misorientation only, without any influence of the local orientation of the grain boundary plane, computational efficiency can be greatly improved by precomputing the possible values of $\gamma$ and $m_{\theta}$ which can occur in the polycrystal. Such a precomputed look-up table is established as a $N_{\phi}$-by- $N_{\phi}$ interface property matrix $A_{\theta}$ where the elements above the diagonal contain the interface energy $\gamma_{i j}$ and the subdiagonal elements contain the misorientation dependence of the interface mobility $m_{\theta, i j}$ for the grain boundary separating grains $i=1, \ldots, N_{\phi}$ and $j=1, \ldots, N_{\phi}$. This storage format is illustrated in Figure 8 . It can be noted that the index $i j$ may be switched arbitrarily, i.e., $(\cdot)_{i j}=(\cdot)_{j i}$. As site-saturated nucleation is considered, all crystal orientations are present from the start and the interface property matrix $A_{\theta}$ can be conveniently established as a preprocessing step with a one-time computational cost. Should subsequent nucleation or grain removal be required, as encountered in dynamic recrystallization, the only added expense is addition and/or removal of singular rows and columns in the matrix. To further optimize memory usage, the diagonal terms of the matrix in Figure 8 can be used to store some other scalar property of the individual grains, such as dislocation density.

\section{NUMERICAL SIMULATIONS}

The model used in the numerical simulations measures $800 \times 200 \mu \mathrm{m}^{2}$ and is shown in Figure 9. The initial model grain structure - comprising 130 grainswas generated by Voronoi tessellation on a square domain which was then compressed along the vertical direction. Different Voronoi tessellations were tested in order to achieve a grain structure which after compression was representative for the actual cold rolled material as observed in the experiments, $c f$. Section II. The microstructure is discretized using triangular elements with linear interpolation and the mesh is updated in each solution step through Delaunay triangularization in order to maintain a high resolution of the grain boundary network. The element size along grain boundaries is controlled by a prescribed maximum element size and a typical mesh is shown in Figure 9. The material parameters used in the level set model are collected in Table I. Following the observations made during the experiments, as discussed in Sections I and II, the initial rolling texture is dominated by the $\alpha$ - and $\gamma$-fibers, with a higher presence of the former. This is in agreement with other experimental studies on rolled IF steel, such as Reference 9. As the $\alpha$ - and $\gamma$-fibers are clearly statistically dominant, the grains in the model microstructure will belong to either of these two populations. To mimic the initially stronger presence of the $\alpha$-fiber, the texture prior to laser annealing is assumed to consist of $60 \mathrm{pct} \alpha$ fiber and 40 pct $\gamma$-fiber. The texture is defined by assigning Euler angles from the appropriate texture distributions with a $5 \mathrm{deg}$ random Gaussian spread around the ideal orientation to the individual grains, providing the initial texture shown in the orientation distribution map in Figure 10(a). The initial texture is also indicated in Figure 9, where $\alpha$-grains are white and $\gamma$-grains are gray. In addition, high-angle grain boundaries - being boundaries with a misorientation greater than $15 \mathrm{deg}$ - are indicated by thicker lines in Figure 9.

The stored energy in 80 pct cold rolled IF steel is measured by neutron diffraction in Reference 53. It is found that, apart from at singular orientations, the $\gamma$ fiber generally has a higher stored energy content compared to the $\alpha$-fiber. Attempts to predict the orientation dependence of the stored energy by means of micromechanical models in Reference 54 show that different results may be found depending on how the mechanical interaction between grains is treated, but a limited influence on the hardening law is obtained. Orientation dependence of the stored energy in lowcarbon steel, for different rolling deformation levels and measured by X-ray diffraction, has been shown in 
Table I. Material Parameters Used in the Numerical Simulations, Representative for IF Steel

\begin{tabular}{llll}
\hline Parameter & \multicolumn{1}{c}{ Value } & \multicolumn{1}{c}{ Description } & Source \\
\hline$b$ & $2.480 \AA$ & magnitude of the Burgers vector & 51 \\
$\mu$ & $69.2 \mathrm{GPa}$ & shear modulus & 14 \\
$\mu$ & $2.66 \times 10^{-19} \mathrm{~J}$ & activation energy for grain boundary migration & 51 \\
$D_{0}$ & $0.89 \times 10^{-4} \mathrm{~m}^{2} / \mathrm{s}$ & grain boundary mobility coefficient & 51 \\
$\gamma_{m}$ & $0.79 \mathrm{~J} / \mathrm{m}^{2}$ & energy of high-angle grain boundaries & 14 \\
$T_{m}$ & $1810 \mathrm{~K}\left(1537^{\circ} \mathrm{C}\right)$ & melting temperature & 51 \\
\hline
\end{tabular}

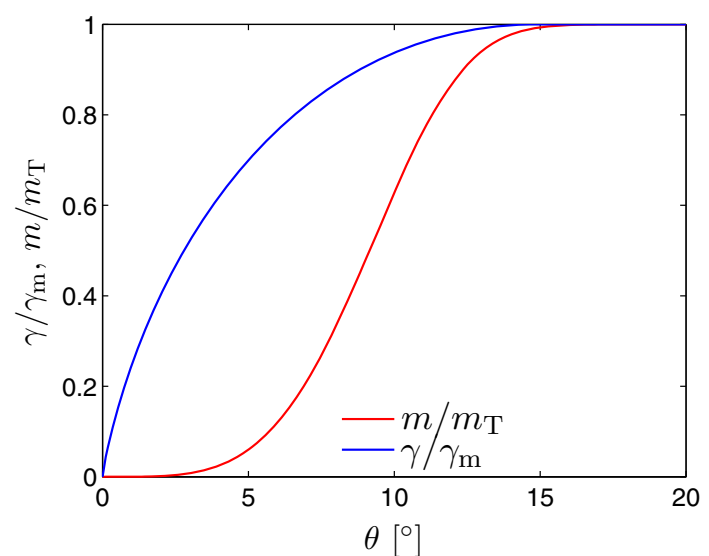

(a)

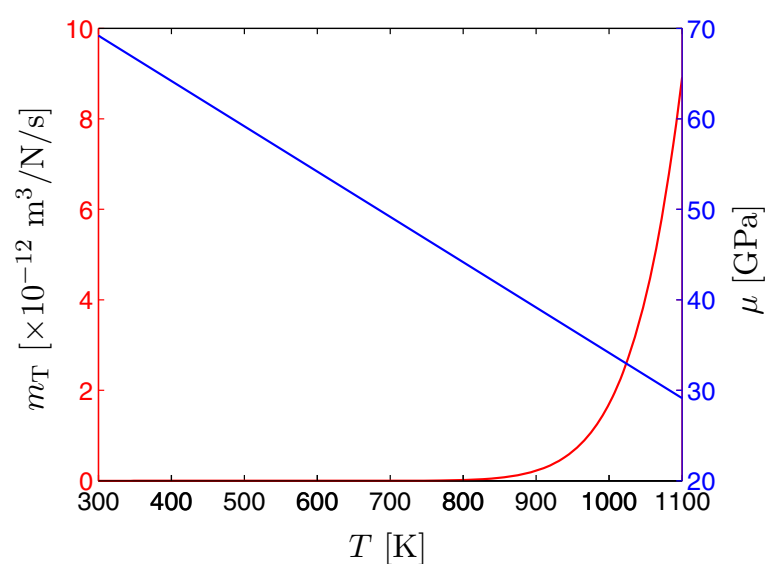

(b)

Fig. 7- (a) Misorientation dependence of the grain boundary mobility and energy, where small-angle boundaries are defined as those having a misorientation $\theta \leq \theta_{m}=15 \mathrm{deg}$. (b) Temperature dependence of the mobility $m_{T}$ for high-angle grain boundaries and for the shear modulus, respectively.

Reference 55. The stored energy levels in IF steel following 75 pct cold rolling are also estimated in Reference 14 and, again, the $\gamma$-fiber is found to have a higher stored energy than the $\alpha$-fiber. The same observation is made in References 56 and 57. Based on these studies, grains belonging to the $\alpha$-fiber are given a dislocation density of $1 \times 10^{15} \mathrm{~m}^{-2}$, while a value of $2 \times$ $10^{15} \mathrm{~m}^{-2}$ is assumed for the $\gamma$-grains in the present work. The stored energy is assumed to be homogeneous in each grain of the $\alpha$ - and $\gamma$-fibers and in the nuclei, respectively. A higher stored energy in the $\gamma$-fiber and a homogeneous stored energy distribution in the grains are also considered in the Monte Carlo Potts model of IF steel in Reference 24 and in the cellular automata model in Reference 14. In Reference 33, it is shown how stored energy gradients can be considered in a level set framework, but this is beyond the scope of the present study. Further, recovery is not considered here as the processing time is very short, only a few seconds. Considerably longer time spans are required for recovery to be of any significance during annealing of IF steel. ${ }^{[56]}$ In passing, it can also be noted that crystal plasticity finite element modeling can be employed to simulate the rolling process and provide an estimate of the stored energy distributions, as discussed in Reference 58 related to IF steel.

Turning to the nucleation, it is widely accepted in the literature that recrystallization during annealing of IF steel primarily nucleates along boundaries of grains in the $\gamma$-fiber after subgrain coarsening. ${ }^{[15]}$ The nuclei are also found to most frequently have their orientations in the same fiber as the parent grain. Similar observations are also made in Reference 59, studying the development of recrystallization textures in pure iron, where recrystallization is found to occur by subgrain growth and grain boundary nucleation with nuclei having approximately $30 \mathrm{deg}$ misorientation with respect to the parent crystals. Regarding IF steel, the study in Reference 17 indicates preferential nucleation of $\gamma$-fiber grains with a $30 \mathrm{deg}$ misorientation around the $\langle 111\rangle / / \mathrm{ND}$ axis. In Reference 20 , it is noted that nucleation in rolled IF steel, being of BCC structure, is most frequent in the $\gamma$ fiber which has a higher stored energy than the $\alpha$-fiber. This is in contrast to most FCC-structured crystals where nucleation rather occurs in texture components with a low stored energy content. Grain boundary nucleation and a dominating nucleation of the $\gamma$-fiber are also observed in Reference 60.

Adopting an approach from studies on FCC materials, the concept of "nucleation factors" is employed in studies on IF steel and low-carbon steel in References 9 and 40. A nucleation factor $N_{i}$ is defined as the number of recrystallized grains of fiber $i$ ( $\alpha$ or $\gamma$ ) per deformation band in the rolled microstructure, observed along ND. This is presented as a measure of the ability of bands of $i$ type to nucleate. Regarding IF steel, the study in Reference 9 presents nucleation factors in the order of 0.1 and 0.8 for $\alpha$ - and $\gamma$-nucleation, respectively, for 
annealing at temperatures of relevance here. These numbers are in the present study adopted as nucleation probabilities for each fiber.

As discussed in Section IV-B, different nucleation and growth conditions will be considered in the simulations, guided by the aforementioned observations. The different simulation scenarios are summarized in Table II. In case 3, the temperature dependence of the grain boundary mobility is kept, while the misorientation dependence of both grain boundary mobility and energy is switched off. In all cases, the initial size of the nuclei is set to $1 \mu \mathrm{m}$, in agreement with the subgrain sizes observed in deformed IF steel in References 8, 18 and 61.

Considering site-saturated nucleation, a prescribed number of nuclei are distributed in the microstructure when initializing the simulations as discussed in Section IV-B. Beginning with nucleation according to case 1, cf. Table II, all possible nucleation sites-i.e., all nodes of the elements containing a grain boundary of a $\gamma$-grain - are identified. Next a number of these possible nucleation nodes are picked randomly, corresponding to the number of nuclei to be placed. In a third step, the nuclei are distributed among these randomly picked sites. When both $\alpha$ - and $\gamma$-nucleation are considered (cases 2 and 3 in Table II), an additional selection step is needed. The nodes of all elements containing the boundaries of both $\alpha$ - and $\gamma$-grains are first identified. But when subsequently distributing the nuclei, the fiber

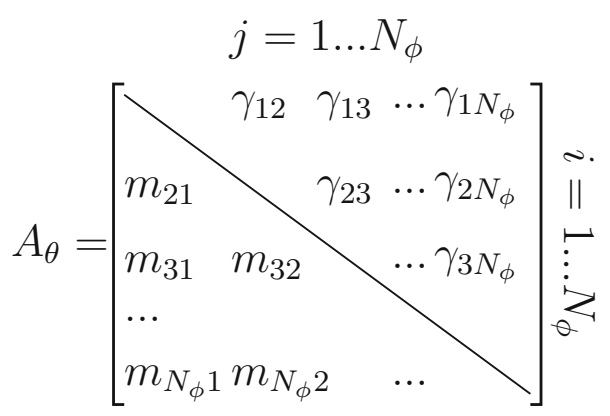

Fig. 8-Layout of the proposed $N_{\phi}$-by- $N_{\phi}$ interface property matrix for precomputed anisotropic grain boundary energy $\gamma_{i j}$ and mobility $M_{\theta, i j}$, related to the grain boundary between crystals $i=1, \ldots, N_{\phi}$ and $j=1, \ldots, N_{\phi}$. The total number of grains present in the polycrystal is denoted by $N_{\phi}$. of the individual nucleation site candidates is identified, after which a random number in the interval $[0,1]$ is generated and compared to the nucleation probability for that fiber ( 0.1 for $\alpha$ and 0.8 for $\gamma$ ). If the random number is less than the nucleation probability, a nucleus is placed at the site, otherwise the site is discarded.

The number of nuclei to place is determined based on estimates of the number of grains and the grain sizes observed in the experiments, for example in Figure 2. The number of nuclei is in this manner controlled to provide annealing microstructure morphologies which correspond to those obtained in the experiments. In the present simulations, 670 new nuclei are seeded in each RVE. Obviously, only those nuclei which have adequate local driving forces will continue to grow as new grains. Starting with 130 cold rolled grains, each RVE will thus comprise 800 grains/nuclei. Some different RVE sizes were investigated but it was found that the relative presence of different texture fibers was quite indifferent to the choice of RVE size. As the relative stability, rather than the absolute presence, of each of the two $\alpha$ and $\gamma$-fibers is under investigation, the adopted grain count and RVE size are assumed sufficient.

\section{RESULTS AND DISCUSSION}

Beginning with the microstructure morphology, Figure 11 shows the appearance of the microstructure in each of the RVEs at the end of the annealing period (35 s), after which the specimen temperature is almost down to its initial value, as shown in Figure 6, and the microstructure has reached a quasi-static state. The results in Figure 11 are found when case 2 in Table II is considered and the results are similar for the other cases. By comparing the RVE microstructures in Figure 11 with the optical microscope image in Figure 1, the same type of gradient in grain size and grain structure can be seen to have developed, with a transition from equiaxed grains in the annealed region towards a maintained laminar rolling structure further out.

In RVE 1 (Figure 11(a)), the recrystallized grain structure is relatively constant throughout the RVE. This is an indication of the small spatial variation in temperature in this particular RVE, located directly

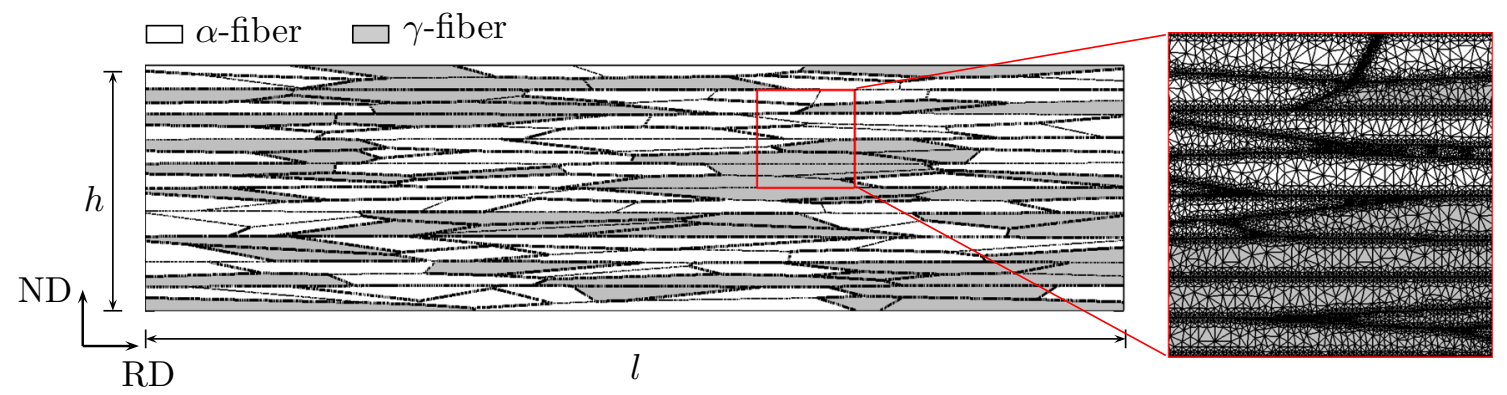

Fig. 9-Initial grain structure of the microstructure RVEs, with a magnification of the typical mesh resolution of the grain boundary network. The initial cold worked microstructure consists of 130 grains. The colors show which texture fiber the grains belong to and thicker grain boundary lines indicate high-angle grain boundaries. The dimensions of the RVE are $l=800 \mu \mathrm{m}$ and $h=200 \mu \mathrm{m}$. The different locations of the RVE in the workpiece are shown in Fig. 5. 
(a) Initial RVE

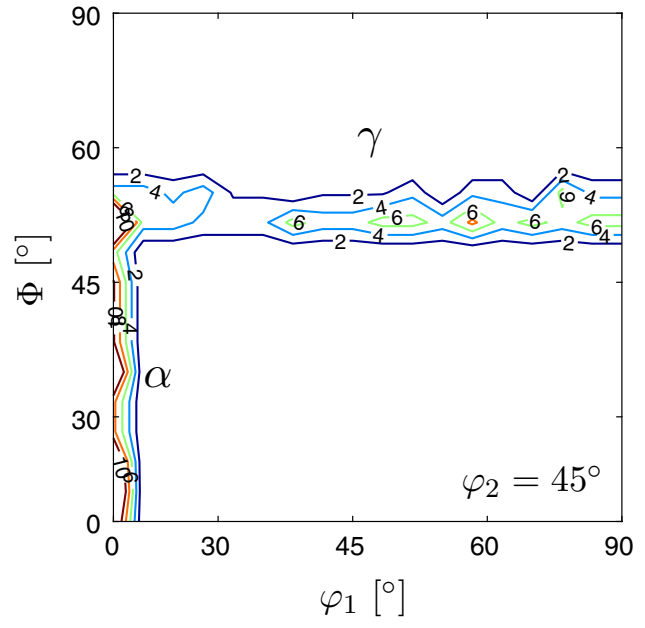

(c) RVE 2, case 2

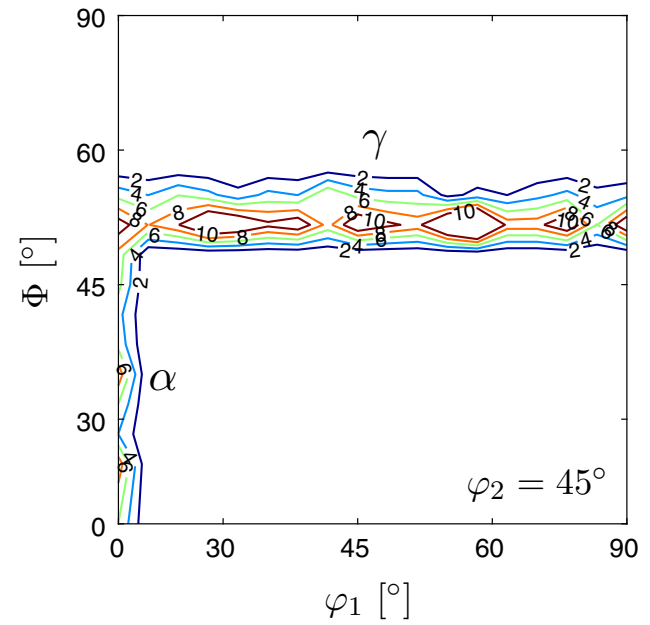

(b) RVE 1, case 2

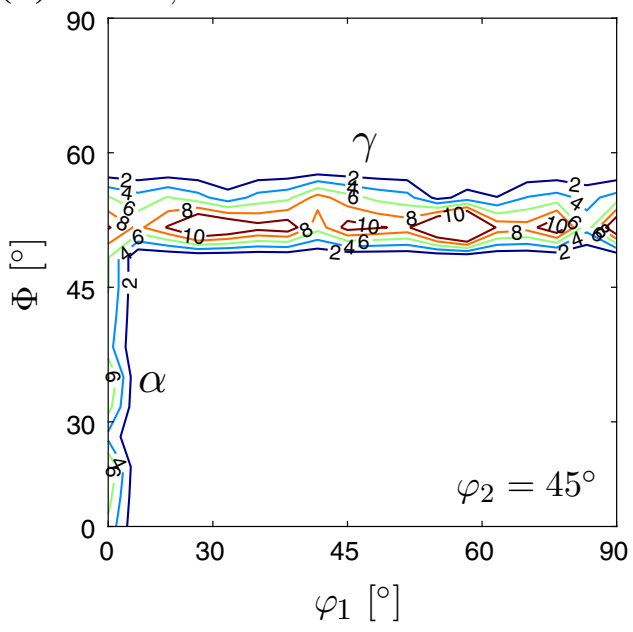

(d) RVE 3, case 2

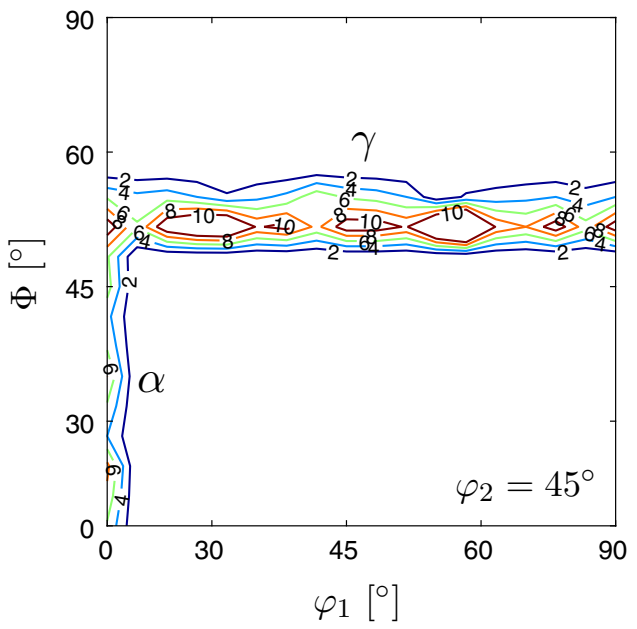

Fig. 10-(a) Initial orientation distribution used in the numerical model, expressed in the Bunge-Euler angles $\left(\varphi_{1}, \Phi, \varphi_{2}\right)$. The texture is assumed to consist only of grains belonging to the dominant $\alpha$ - and $\gamma$-fibers, as shown in Fig. 9. Figures $(b)$ through $(d)$ show the orientation distribution after annealing according to case 2, cf. Table II, for the different RVEs: (b) RVE 1, (c) RVE 2, and (d) RVE 3. In all cases, the $\gamma$-fiber is strengthened at the expense of the $\alpha$-fiber after annealing. The same isolines (intensity levels 2, 4, 6, 8, and 10) are plotted in all figures.

Table II. Simulation Scenarios Investigated in the Numerical Simulations

\begin{tabular}{ll}
\hline Case & \multicolumn{1}{c}{ Nucleation and growth conditions } \\
\hline 1 & nucleation only of $\gamma$-grains. Nuclei get a random orientation from the $\gamma$-fiber \\
2 & nucleation of both $\alpha$ - and $\gamma$-grains with different probabilities. Nuclei get a random orientation from the parent fiber. \\
3 & nucleation of both $\alpha$ - and $\gamma$-grains with different probabilities. Nuclei get a random orientation from the parent fiber. \\
& The misorientation dependence of the grain boundary energy and mobility is switched off $\left(m=m_{T}\right.$ and $\left.\gamma=\gamma_{m}\right)$ \\
\hline
\end{tabular}

beneath the laser beam center. The small temperature variation is also seen in the nearly coinciding temperature graphs for RVE 1 in Figure 6. The gradient becomes steeper as RVE 2 and RVE 3 are considered, manifested by a greater variation in the recrystallization behavior in the rolling direction in Figures 11(b) and (c). Only a small number of recrystallization nuclei have started to grow at the high-temperature left end of RVE 3, which is located at a distance of $4 \mathrm{~mm}$ away from the laser beam centerline. This is in agreement with the hardness mapping and grain size measurements in Figures 3 and 4, respectively, showing that the microstructure is relatively unaffected by the laser annealing in this region.

As anisotropic grain boundary properties are considered, i.e., when grain boundary energy and mobility are allowed to depend on the misorientation, the local grain growth kinetics will vary and grains may exhibit protrusions and irregularities. Figure 12(a) illustrates this by showing a region in RVE 1 (case 2) where 
(a) RVE1 $\square \alpha$-fiber $\square \gamma$-fiber

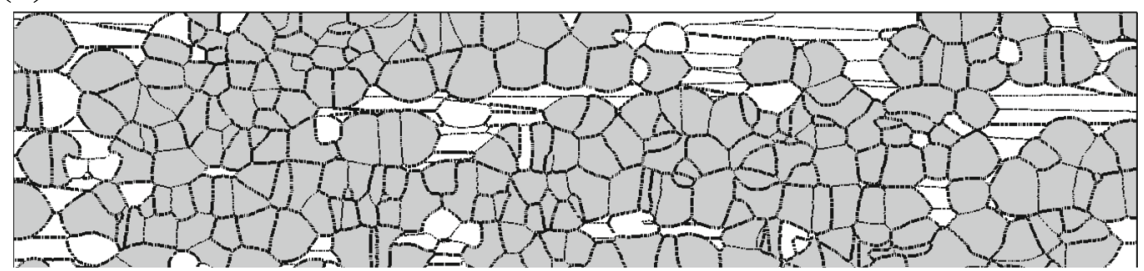

(b) RVE2

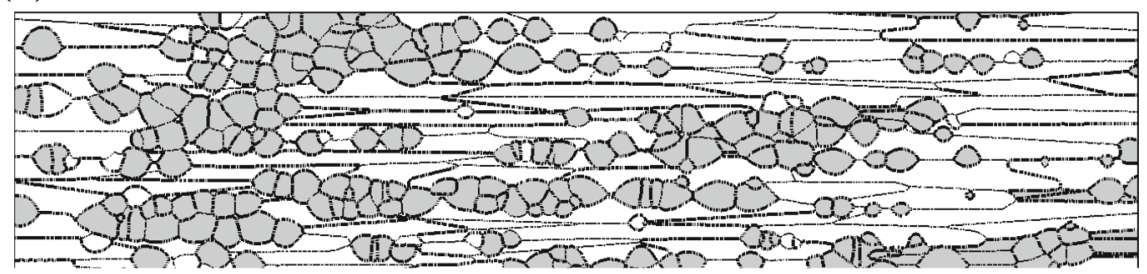

(c) RVE3

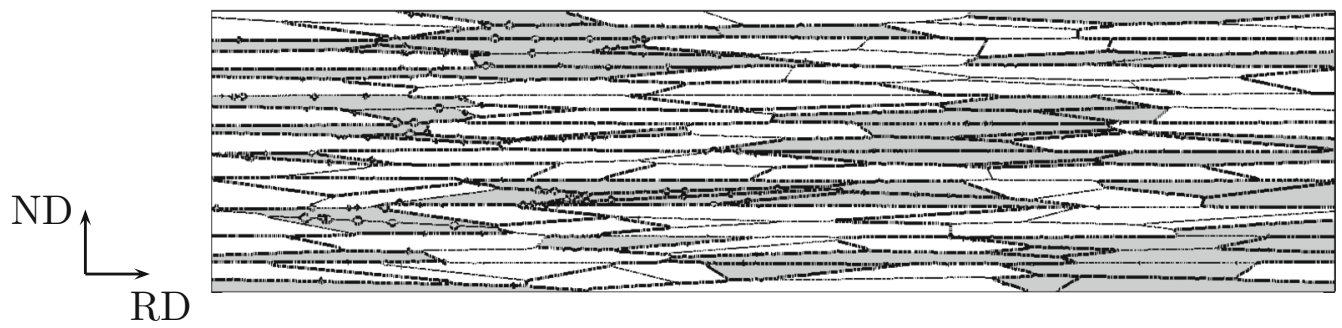

Fig. 11-Microstructure after laser annealing. The figures show the different RVEs indicated in Fig. 5 and located at different distances $r$ from the laser beam centerline. Grains in the $\alpha$ - and $\gamma$-fibers are white and gray, respectively, and high-angle grain boundaries are drawn in thicker lines than low-angle boundaries. (a) RVE $1(0 \leq r \leq 0.8 \mathrm{~mm})$, (b) RVE $2(2 \leq r \leq 2.8 \mathrm{~mm})$, and (c) RVE $3(4 \leq r \leq 4.8 \mathrm{~mm})$.

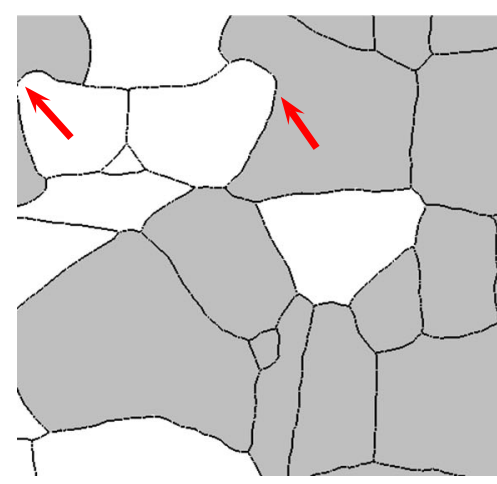

(a)

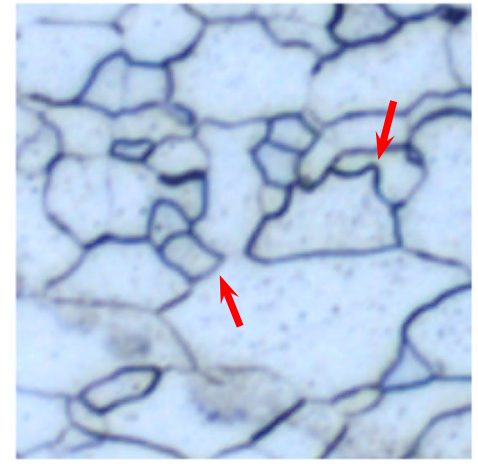

(b)

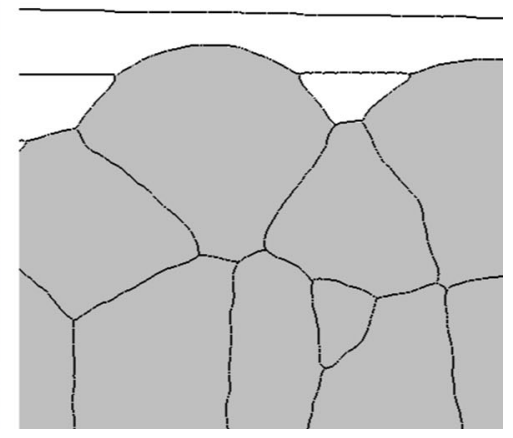

(c)

Fig. 12-(a) Irregular protrusions of the grain boundaries, indicated by the arrows, as seen in a simulated microstructure when anisotropic grain boundary properties are considered (RVE 1, case 2). (b) Similar grain features observed by optical microscopy. (c) Grain boundaries without irregularities in the case of isotropic grain boundary properties (RVE 1, case 3).

recrystallization grains have grown under locally varying grain boundary conditions. Similar microstructural features are also observed in the optical microscope image shown in Figure 12(b), taken from the laser annealing experiments. Growth of elongated grains and small grain protrusions is also found, for example, in the Monte Carlo Potts modeling studied in Reference 62. If isotropic grain boundary properties are assumed, i.e., $m=m_{T}$ and $\gamma=\gamma_{m}$ (case 3), the grains will grow with boundaries forming circular arcs, except for where triple junction influence strives to maintain the standard 120 deg equilibrium configuration between connecting grain boundaries. This is illustrated in Figure 12(c), where a region in RVE 1 (case 3) exhibits approximately circular grain boundary lines.As shown in Figure 11(a), the last regions to be consumed by recrystallization are the cold worked $\alpha$-grains, consistent with observations made during the laser annealing experiments and also with the findings in References 14 and 19. Since the dislocation density is higher in the 
(a) RVE 1

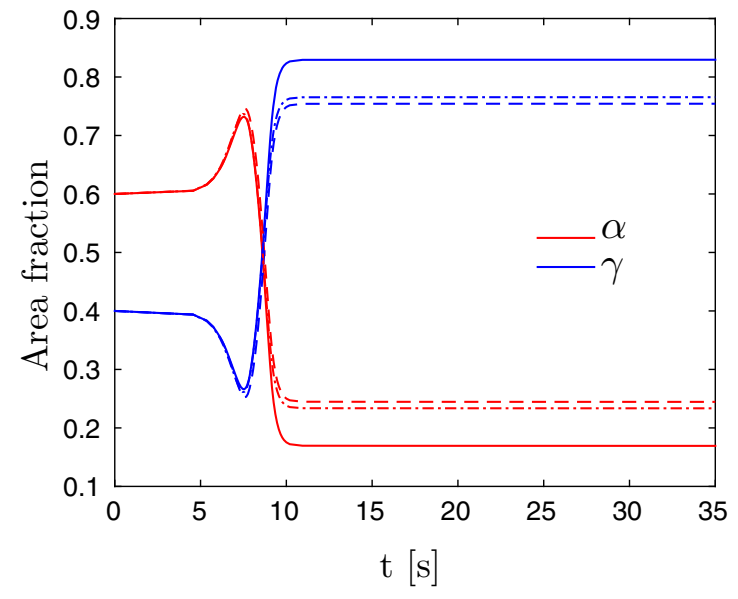

(b) RVE 2

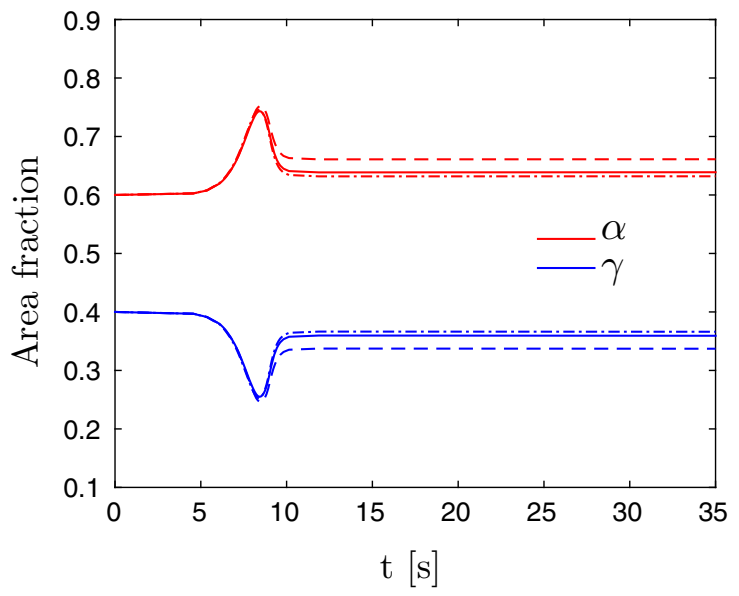

(c) RVE 3

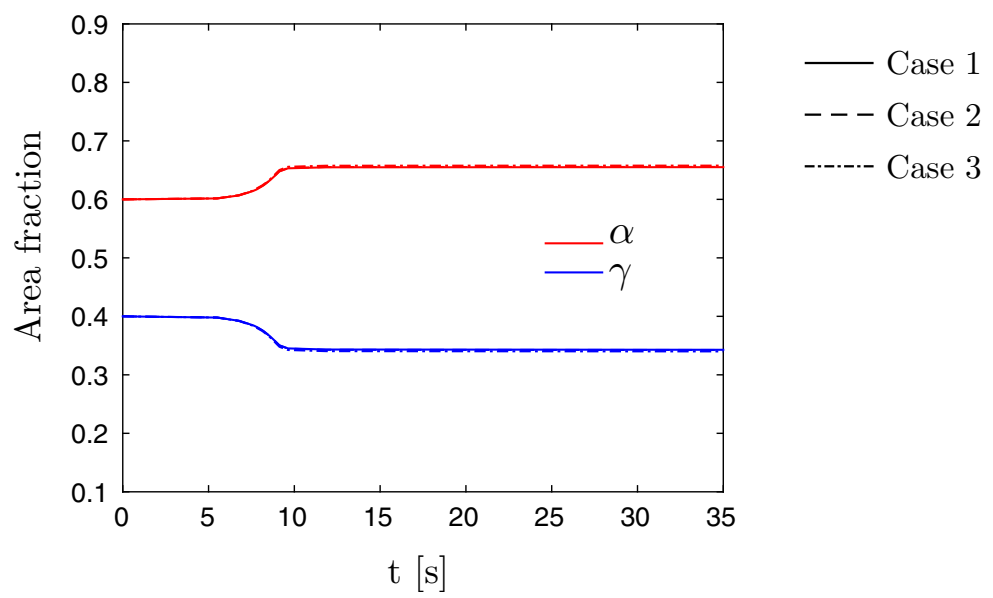

Fig. 13 -Evolution of the area fractions of the $\alpha$ - and $\gamma$-fibers in the different RVEs during laser annealing. The line styles indicate the different cases defined in Table II: case $1=$ solid line, case $2=$ dashed line, and case $3=$ dash-dotted line. (a) RVE 1 , $(b)$ RVE 2 , and (c) RVE 3.

$\gamma$-fiber, the growth rates are higher there compared to those in $\alpha$-regions, having a relatively lower dislocation content. ${ }^{[56]}$ This results in the cold worked $\gamma$-fiber being more rapidly transversed by the recrystallization front. The initial microstructure is assumed to have a dominance of 60 pet $\alpha$-fiber over 40 pet $\gamma$-fiber, following observations made in the experiments. This means that there will be a frequency/size advantage of the $\alpha$-fiber. However, nucleation is significantly more frequent in the $\gamma$-fiber, giving this fiber a nucleation advantage that is seen to be critical. The initial texture is shown in the orientation distribution plot in Figure 10(a). Correspondingly, Figures 10(b) through (d) show the orientation distribution in the different RVEs after annealing, assuming case 2 as defined in Table II. It can be noted that the $\gamma$-fiber is strengthened in all RVEs, while the $\alpha$-fiber at the same time is weakened. This is in agreement with, for example Reference 17, where annealing is found to nearly annihilate the $\alpha$-fiber entirely, except for at the orientation $(\{111\}\langle 110\rangle)$, which is common to both fibers.

Another view of the evolution of the texture fibers is provided in Figure 13, showing the area fractions of the $\alpha$ - and $\gamma$-fibers throughout the annealing process in all RVEs and for all simulation cases. Initially, the $\alpha$-fiber increases while the $\gamma$-fiber decreases. This occurs during the heating phase of the annealing process as the deformed grain boundaries in the cold worked microstructure become increasingly mobile and since the $\alpha$-fiber has a growth advantage over the $\gamma$-fiber due to a lower stored energy. But as sufficient temperature levels are reached, the nuclei attain sufficient grain boundary mobility to expand, which occurs very rapidly under a strong stored energy driving force. The tendency in the evolution of the fiber area fractions is now reversed in RVE 1 and RVE 2-Figures 13(a) and (b)as growing $\gamma$-nuclei consume the $\alpha$-fiber. If recrystallization would be complete, the $\alpha$-fiber in RVE 1 would be entirely annihilated. However, while the temperature is sufficiently high in RVE 1 to make the $\gamma$-fiber completely dominant, the duration of the high-temperature peak in RVE 2 is too short to allow the growing nuclei to provide dominance to the $\gamma$-fiber. This is even more pronounced when considering RVE 3 in Figure 13(c), where the $\alpha$-fiber maintains its stronger presence throughout the entire process. Figure 13 gives a strong 
(a) RVE 1

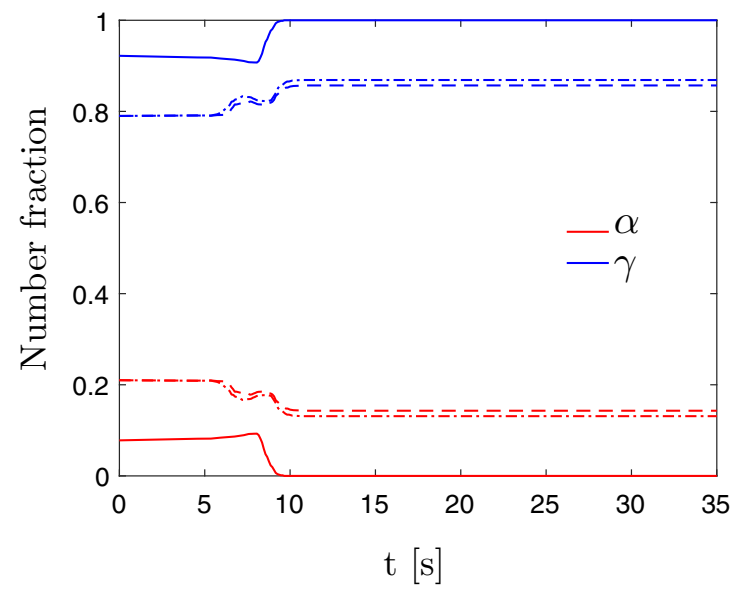

(b) RVE 2

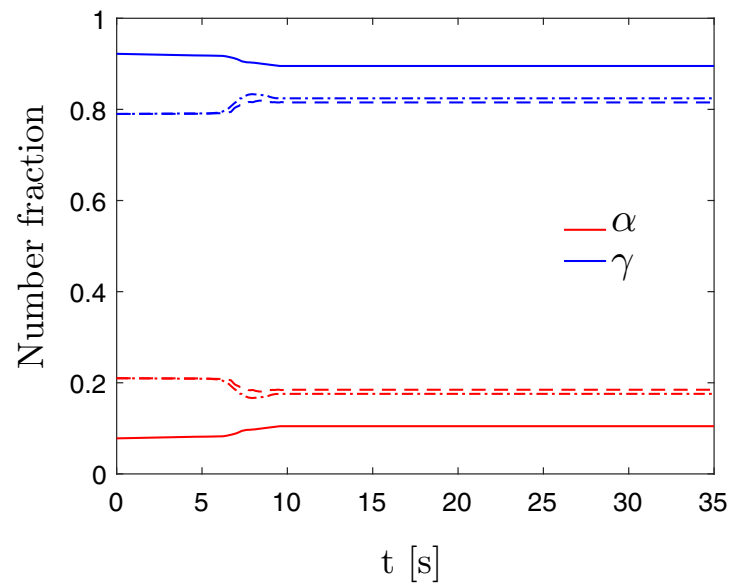

(c) RVE 3

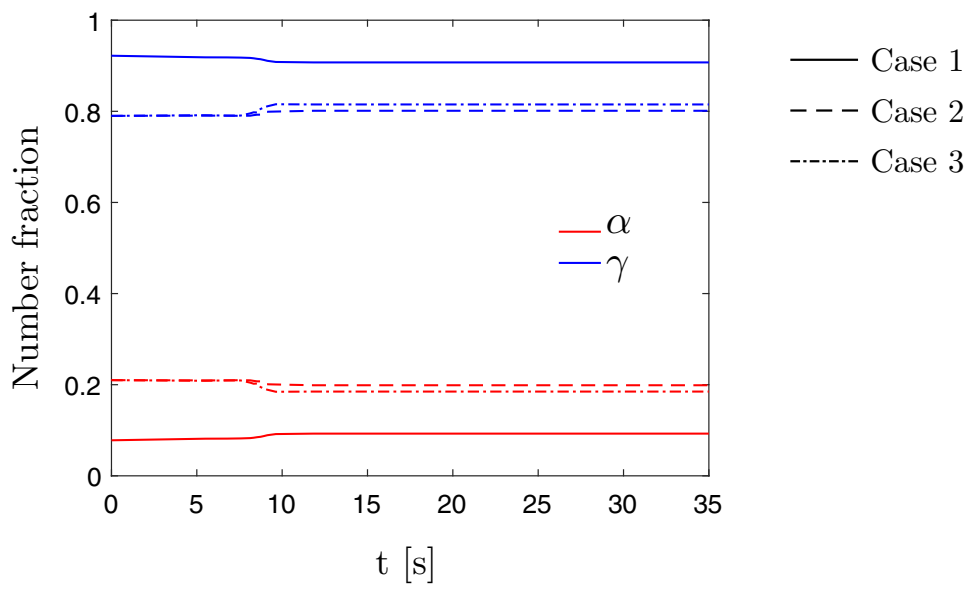

Fig. 14-Evolution of the number fractions of grains belonging to the $\alpha$ - and $\gamma$-fibers, respectively, in the different RVEs during laser annealing. The line styles indicate the different cases defined in Table II: case $1=$ solid line, case $2=$ dashed line, and case $3=$ dash-dotted line. (a) RVE 1 , (b) RVE 2, and (c) RVE 3.

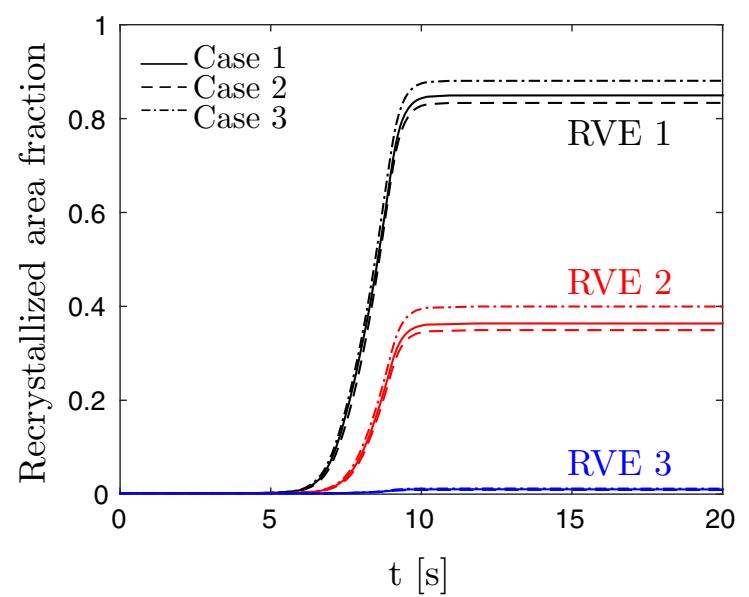

(a)

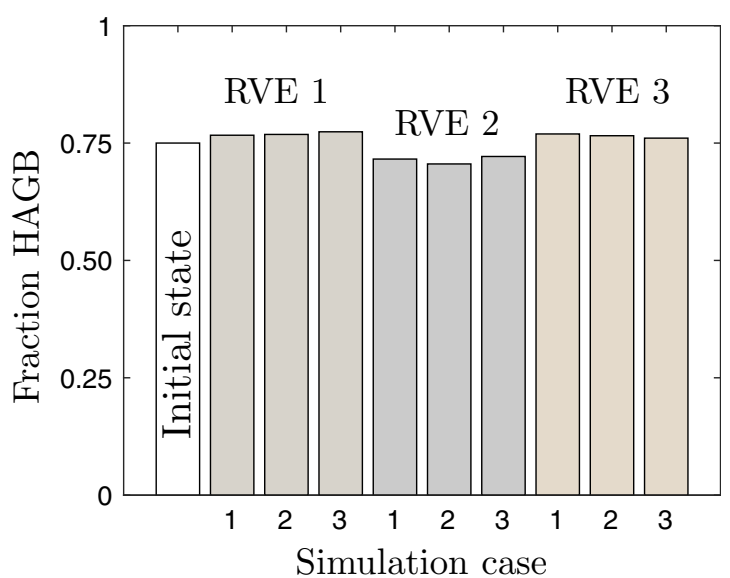

(b)

Fig. 15- (a) Recrystallized area fraction $v s$ time for the different simulation scenarios. The line color indicates the RVE and the line type which case, $c f$. Table II. (b) Length-weighted fraction of high-angle grain boundaries (HAGB) at the end of the different annealing scenarios. 
indication that the final annealing texture will depend greatly on which region in the annealing zone one chooses to observe.Considering the orientation distribution functions (ODFs) shown in Figure 10, the strength of the $\gamma$-fiber is apparently increased in all three simulation cases. But the ODFs are based on the fiber presence in orientation space and not in terms of area fraction as in Figure 13. Hence, even very small $\gamma$ grains will make a contribution towards a more pronounced $\gamma$-fiber in the ODFs, while the area fractions in Figure 13 will not show any appreciable strengthening of the $\gamma$-fiber. This point is further illustrated in Figure 14, showing the number fraction of grains belonging to each texture fiber. Comparing Figures 13 and 14, it can, for example, be observed that the area fraction of $\gamma$-grains may be lower than the area fraction of $\alpha$-grains, while the opposite relation holds for the number fractions. It can also be noted, in both Figures 13 and 14, that the most significant changes take place at a time of 9 seconds, when the peak temperature is reached and the laser is switched off.

As noted in the introduction, a strong presence of the $\gamma$-fiber is related to a significantly enhanced ductility in the material. This property will also vary according to the observations made here.

It can be noted that the stored energy in the grains will vary depending on the crystal orientations, often explained in terms of the Taylor factor. A lower stored energy content will be found in the $\alpha$-fiber compared to the $\gamma$-fiber, $c f .{ }^{[56]}$ This means that the dislocation substructures necessary for nucleation of new grains are more likely to be found in grains with orientations in the $\gamma$-fiber. This provides a partial explanation for the preferential growth of the $\gamma$-fiber, as discussed in References 44, 59 and 63. Another factor to influence the growth kinetics is the availability of grain boundaries with high mobility, generally being high-angle boundaries. This is also seen to work in favor of the $\gamma$ fiber, as noted in Reference 23. In the presence of strong stored energy driving forces, it is, however, expected that the variations in grain boundary mobility will only have a minor influence on the selective growth behavior compared to the stored energy. ${ }^{[20,64]}$

The progress of recrystallization is shown in Figure 15 (a) in terms of the recrystallized area fraction as a function of time. Again, all RVEs and all simulation cases are shown. It can be seen that the particular case being adopted has a relatively small impact on the recrystallization kinetics (the slope of the curve), but a larger influence on the final recrystallized area fraction. Only considering nucleation of the $\gamma$-fiber is seen to slightly overestimate the recrystallized fraction compared to case 2 in which nucleation of both fibers is accounted for. But most significantly, if anisotropy of the grain boundary properties is not accounted for (case 3), the recrystallized fraction-and hence also the presence of the $\gamma$-fiber-is overestimated by approximately 6 pct. As noted previously, the temperatures reached in RVE 3, cf. Figure 15(c), are insufficient to provide the grain boundary mobility required for any significant recrystallization to take place.
Figure 15 shows the length-weighted fractions of high-angle grain boundaries (HAGB) - taken to be those boundaries having a misorientation greater than $15 \mathrm{deg}$ - in the microstructure before and after laser annealing. The variations are small, but it can be noted that the fraction of HAGB increases slightly in both RVE 1 and RVE 3, while a minor decrease in the HAGB fraction is seen in RVE 2 for all cases considered. In RVE 1, a randomization of the grain boundary texture has taken place due to the almost complete recrystallization and in RVE 3 the initial grain boundary network is nearly intact with the exception of a few slightly expanded nuclei. In RVE 2, most of the $\gamma$-fiber has been recrystallized, leaving mainly low-angle boundaries in the remaining $\alpha$-fiber. These different situations are also shown in Figure 11, where HAGB are drawn with thicker lines compared to LAGB.

\section{CONCLUDING REMARKS}

Laser annealing has been found to be a technique that holds much potential for manufacturing of functionally graded metal components, and the proposed modeling approach provides a tool for optimizing the process by linking processing conditions to microstructure evolution. The simulations are performed in a finite elementbased level set framework and account for anisotropic, $i$. e., misorientation-dependent, grain boundary properties in terms of energy and mobility. The strong variation of mobility with temperature is also considered. In addition, the model considers the development of recrystallization texture due to nucleation and grain growth in the presence of stored energy driving forces and a transient thermal gradient caused by the local laser irradiation. As such, the model incorporates the central aspects of the microstructure development during laser annealing and the simulation results are found to agree well with experimental observations.

The numerical simulation model is used to investigate a number of phenomena which are active in the microstructure during laser annealing. In particular, the influence of different nucleation and growth conditions on the evolution of the grain structure and of the typical two-fiber recrystallization texture is studied.

It is found that the initial dominance of the $\alpha$-fiber is at first emphasized as the boundaries of the cold worked grains become increasingly mobile with increasing temperature. This tendency is driven by a higher stored energy content in the $\gamma$-fiber. At locations in the annealing zone where the temperature is sufficient, expanding recrystallization nuclei will reverse the trend and gradually consume the $\alpha$-fiber as recrystallization progresses. In regions of lower temperature, however, the $\alpha$-fiber will maintain and even increase its dominance. The $\gamma$-fiber is desirable as it increases the formability of the material, and the extent of the $\gamma$ dominated zone that develops for certain laser process settings can be estimated by numerical simulations as the ones performed here. This was shown by comparing the simulation results with measurements of the varia- 
tions of hardness and grain size across the annealing zone.

Plots of the orientation distributions show that the simulations capture the growth of the $\gamma$-fiber at the expense of the $\alpha$-fiber. This is, however, not a general trend but depends on where in the thermal gradient the observation is made.

Selective growth is in the present model included by considering the misorientation dependence of grain boundary energy and mobility, meaning that the anisotropy is characterized by three parameters (the Euler angles) only. Although a full description of the grain boundary characteristics requires a five-parameter formulation (three Euler angles plus two parameters to describe the inclination of the grain boundary plane), the present model should be sufficient to provide an indication of the influence of anisotropic grain boundary properties, as noted in, for example, References 65 through 68. The anisotropic grain boundary properties are found to have a relatively limited influence on the recrystallization kinetics. In contrast, the final recrystallized fraction will be clearly overestimated if the misorientation dependence is neglected. In addition, without anisotropic grain boundary properties, the nuclei will expand with approximately circular grain boundary fronts, while the local grain boundary irregularities observed in experiments require anisotropic grain boundary conditions to be considered. It can also be noted that a heterogeneous stored energy distribution within individual grains can be expected to exert additional influence on the morphology of expanding grains. This has, however, not been considered in the present study as a homogeneous stored energy has been assumed in each grain.

In the present simulations, stored energy driving forces are essential for growth of the recrystallization nuclei. The fiber in which the nucleation takes place is also seen to be a highly influential factor. Although the $\alpha$-fiber has a frequency advantage initially, the nucleation advantage of the $\gamma$-fiber, having a higher stored energy, promotes recrystallization to primarily consume the cold worked $\gamma$ fiber and leave the $\alpha$-fiber to be recrystallized last. These observations are in agreement with the experimental data and with observations found in the literature.

In Reference 36, the nucleation mechanism is in general found to be more influential than the growth mechanism in determining the recrystallization microstructure. This is, however, further complicated in the case of local laser annealing as microstructure evolution takes place in a significant and transient thermal gradient. Thereby, growth conditions in terms of a temperature-dependent grain boundary mobility come to play a much more central role than what is expected in the isothermal case.

\section{ACKNOWLEDGMENTS}

H. Hallberg gratefully acknowledges funding from Ecole Nationale Supérieure d'Arts et Métiers (ENSAM) at ParisTech. All the authors are grateful to Patrice
Peyre and Fréderic Costes at ENSAM for their assistance in the laser annealing experiments and A. Perlade at ArcelorMittal for supplying the steel material for the experimental investigations.

\section{OPEN ACCESS}

This article is distributed under the terms of the Creative Commons Attribution 4.0 International License (http://creativecommons.org/licenses/by/4.0/), which permits unrestricted use, distribution, and reproduction in any medium, provided you give appropriate credit to the original author(s) and the source, provide a link to the Creative Commons license, and indicate if changes were made.

\section{REFERENCES}

1. J. Pröll, R. Kohler, M. Torge, S. Ulrich, C. Ziebert, M. Bruns, H. J. Seifert, and W. Pfleging: Appl. Surf. Sci., 2011, vol. 257, pp. 9968-76

2. C. Charpentier, R. Boukhicha, P. Prod'homme, T. Emeraud, J.-F. Lerat, P. Roca i Cabarrrocas, and E.V. Johnson: Sol. Energy Mater. Sol. Cells, 2014, vol. 125, pp. 223-32.

3. S.Y. Lee, E.S. Shim, H.S. Kang, S.S. Pang, and J.S. Kang: Thin Solid Films, 2005, vol. 473, pp. 31-34.

4. Y. Inaba, T.J. Klemmer, Y. Kubota, J.W. Harrell, and G.B. Thompson: Thin Solid Films, 2012, vol. 524, pp. 278-81.

5. Q. Meng, Y. Liu, H. Yang, B.S. Shariat, and T. Nam: Acta Mater., 2012, vol. 60 , pp. $1658-68$.

6. L.W. Tsay, W.C. Lee, W.C. Luu, and J.K. Wu: Corros. Sci., 2002, vol. 44, pp. 1311-27.

7. L.W. Tsay, Y.C. Liu, D.-Y. Lin, and M.C. Young: Mater. Sci. Eng. A, 2004, vol. 384, pp. 177-83.

8. I. Thomas, S. Zaefferer, F. Friedel, and D. Raabe: Adv. Eng. Mat., 2003 , vol. 5 , pp. 566-70.

9. I. Samajdar, B. Verlinden, and P. Van Houtte: Acta Mater., 1998, vol. 46, pp. 2751-63.

10. D. Raabe and K. Lücke: Mater. Sci. Forum, 1994, vol. 157-162, pp. 597-610.

11. T. Urabe and J.J. Jonas: ISIJ Int., 1994, vol. 34, pp. 435-42.

12. W.B. Hutchinson: Int. Mater. Rev., 1984, vol. 29, pp. 25-42.

13. M.Z. Quadir and B.J. Duggan: Acta Mater, 2004, vol. 52, pp. 401121.

14. D. Raabe and L. Hantcherli: Comput. Mater. Sci., 2005, vol. 34, pp. 299-313

15. R.K. Ray, J.J. Jonas, and R.E. Hook: Int. Mater. Rev., 1994, vol. 39 , pp. $129-71$

16. C.S. He, Y.D. Zhang, Y.N. Wang, X. Zhao, L. Zuo, and C. Esling: Scr. Mater, 2003, vol. 48, pp. 737-42.

17. M.Z. Quadir and B.J. Duggan: Acta Mater., 2006, vol. 54, pp. $4337-$ 50 .

18. A. Samet-Meziou, A.L. Etter, T. Baudin, and R. Penelle: Scr. Mater., 2005, vol. 53, pp. 1001-06.

19. Y.Y. Tse and B.J. Duggan: Metall. Mater. Trans. A, 2006, vol. 37A, pp. $1055-64$.

20. I. Samajdar, B. Verlinden, P. Van Houtte, and D. Vanderschueren: Mater. Sci. Eng. A, 1997, vol. 238, pp. 343-50.

21. M. Hölscher, D. Raabe, and K. Lücke: Steel Res., 1991, vol. 62, pp. $567-75$.

22. K. Wierzbanowski, J. Tarasiuk, B. Bacroix, A. Miroux, and O. Castelnau: Arch. Metall., 1999, vol. 44, pp. 183-201.

23. L. Kestens and J.J. Jonas: Metall. Mater. Trans. A, 1996, vol. 27A, pp. $155-64$

24. S.-H. Choi, F. Barlat, and J.H. Chung: Scr. Mater, 2001, vol. 45, pp. $1155-62$.

25. S.-H. Choi and J.H. Cho: Mater. Sci. Eng. A, 2005, vol. 405, pp. 86101. 
26. S. Osher and J.A. Sethian: J. Comput. Phys., 1988, vol. 79, pp. 12-49.

27. A. Agnoli, N. Bozzolo, R. Logé, J.-M. Franchet, J. Laigo, and M. Bernacki: Comput. Mater. Sci., 2014, vol. 89, pp. 233-41.

28. A.L. Cruz-Fabiano, R. Logé, and M. Bernacki: Comput. Mater. Sci., 2014, vol. 92, pp. 305-12.

29. M. Elsey, S. Esedoglu, and P. Smereka: J. Comput. Phys., 2009, vol. 228, pp. 8015-33.

30. M. Elsey, S. Esedoglu, and P. Smereka: Acta Mater, 2013, vol. 61, pp. 2033-43.

31. H. Hallberg: Modell. Simul. Mater. Sci. Eng., 2014, vol. 22, p. 085005 .

32. M. Bernacki, Y. Chastel, T. Coupez, and R.E. Logé: Scr. Mater, 2008, vol. 58, pp. 1129-32.

33. H. Hallberg: Modell. Simul. Mater. Sci. Eng., 2013, vol. 21, p. 085012.

34. R. Logé, M. Bernacki, H. Resk, L. Delannay, H. Digonnet, Y. Chastel, and T. Coupez: Philos. Mag., 2008, vol. 88, pp. 3691-3712.

35. H. Hallberg: Metals, 2011, vol. 1, pp. 16-48.

36. R. Sebald and G. Gottstein: Acta Mater, 2002, vol. 50, pp. 1587-98.

37. S. Mishra and T. DebRoy: Acta Mater, 2004, vol. 52, pp. 1183-92.

38. M. Toloui and M. Militzer: Int. J. Mater. Res., 2010, vol. 101, pp. $542-48$.

39. V. Tikare and E.A. Holm: J. Am. Ceram. Soc., 1998, vol. 81, pp. 480-84.

40. I. Samajdar, B. Verlinden, L. Kestens, and P. Van Houtte: Acta Mater, 1999, vol. 47, pp. 55-65.

41. L. Costa, R. Vilar, T. Reti, and A.M. Deus: Acta Mater, 2005, vol. 53, pp. 3987-99.

42. M.C. Teich and B.E.A. Saleh: Fundamentals of Photonics, Wiley, New York, 1991

43. I.A. Roberts, C.J. Wang, R. Esterlein, M. Stanford, and D.J. Mynors: Int. J. Mach. Tool. Manuf., 2009, vol. 49, pp. 916-23.

44. F.J. Humphreys and M Hatherly: Related Annealing Phenomena, Second ed., Pergamon, New York, 2004.

45. R.D. Doherty, D.A. Hughes, F.J. Huphreys, J.J. Jonas, D. Juul Jensen, M.E. Kassner, W.E. King, T.R. McNelley, H.J. McQueen, and A.D. Rollett: Mater. Sci. Eng. A, 1997, vol. 238, pp. 219-74.

46. F.J. Humphreys: Acta Mater, 1997, vol. 45, pp. 4231-40.

47. F.J. Humphreys: Mater. Sci. Technol., 1992, vol. 8, pp. 135-44.

48. B.L. Li, A. Godfrey, Q.C. Meng, Q. Liu, and N. Hansen: Acta Mater, 2004, vol. 52, pp. 1069-81.
49. D.J. Srolovitz, G.S. Grest, and M.P. Anderson: Acta Metall., 1986, vol. 34, pp. 1833-45.

50. D.J. Srolovitz, G.S. Grest, M.P. Anderson, and A.D. Rollett: Acta Metall., 1988, vol. 36, pp. 2115-28.

51. H.J. Frost and M.F. Ashby: Deformation Mechanism Maps: The Plasticity and Creep of Metals and Ceramics, Pergamon Press, Oxford, 1982.

52. M. Militzer, W.P. Sun, and J.J. Jonas: Acta Metall. Mater., 1994, vol. 42, pp. 133-41.

53. N. Rajmohan, Y. Hayakawa, J.A. Szpunar, and J.H. Root: Acta Mater, 1997, vol. 45, pp. 2485-94.

54. B. Bacroix, A. Miroux, and O. Castelnau: Modell. Simul. Mater. Sci. Eng., 1999, vol. 7, pp. 851-64.

55. A. Wauthier-Monnin, T. Chauveau, O. Castelnau, H. Réglé, and B. Bacroix: Mater. Charact., 2015, vol. 104, pp. 31-41.

56. M. Oyarzábal, A. Martínez de Guerenu, and I. Gutıerrez: Mater. Sci. Eng. A, 2008, vol. 485, pp. 200-09.

57. D. Vanderschueren. In: Tokyo: The Iron and Steel Institute of Japan, 1994, p. 145.

58. S.-H. Choi: Acta Mater., 2003, vol. 51, pp. 1775-88.

59. W.B. Hutchinson: Acta Metall., 1989, vol. 37, pp. 1047-56.

60. Y. Hayakawa and J.A. Szpunar: Acta Mater., 1997, vol. 45, pp. 3721-30.

61. B.L. Li, W.Q Cao, Q. Liu, and W. Liu: Mater. Sci. Eng. A, 2003, vol. 356 , pp. $37-42$.

62. K. Okuda and A.D. Rollett: Comput. Mater. Sci., 2005, vol. 34, pp. 264-73.

63. S.-H. Choi and Y.-S. Jin: Mater. Sci. Eng. A, 2004, vol. 371, pp. 149-59.

64. H. Hallberg and P.A.T. Olsson: J. Mech. Phys. Solids, 2016, vol. 90, pp. 160-78.

65. G.N. Hassold, E.A. Holm, and M.A. Miodownik: Mater. Sci. Technol., 2003, vol. 19, pp. 683-87.

66. D.C. Hinz and J.A. Szpunar: Phys. Rev. B, 1995, vol. 52, pp. 9900 09.

67. E.A. Holm, G.N. Hassold, and M.A. Miodownik: Acta Mater, 2001, vol. 49, pp. 2981-91.

68. A. Kazaryan, Y. Wang, S.A. Dregia, and B.R. Patton: Acta Mater, 2002, vol. 50, pp. 2491-2502. 